\title{
CATÁLOGO DE LAS ESPECIES DE SABELLIDAE LATREILLE, 1825 Y FABRICIIDAE RIOJA, 1923 (ANNELIDA, SABELLIDA) CITADAS EN LA PENÍNSULA IBÉRICA E ISLAS BALEARES Y CHAFARINAS
}

\author{
Eduardo López ${ }^{1 *} \&$ Diego Cepeda ${ }^{2}$ \\ ${ }^{1}$ Departamento de Biología, Universidad Autónoma de Madrid, C/ Darwin, 2, 28049-Madrid. \\ ORCID iD: http://orcid.org/0000-0002-1816-8528 \\ ${ }^{2}$ Departamento de Zoología y Antropología Física, Universidad Complutense de Madrid, C/ José Antonio Novais, 12 , \\ 28040-Madrid. ORCID iD: http://orcid.org/0000-0002-5194-3346 \\ * Autor para correspondencia: e-mail: eduardolopezuam@gmail.com
}

\section{RESUMEN}

Se ha elaborado un listado taxonómico comentado de los sabelidos y fabrícidos de la Península lbérica e Islas Chafarinas y Baleares a partir de la revisión exhaustiva de la bibliografía publicada referida directa o indirectamente a estos grupos. En total se han reportado 54 especies válidas incluidas en 23 géneros pertenecientes a Sabellidae y nueve especies pertenecientes a siete géneros de Fabriciidae. En esta lista se incluyen para cada especie todas las citas para el área estudiada, así como los sinónimos utilizados en las mismas e información sobre su distribución geográfica a nivel regional.

Palabras clave: Sabellidae; Fabriciidae; Polychaeta; Fauna lbérica; Catálogo.

\section{ABSTRACT}

Checklist of the species of Sabellidae Latreille, 1825 and Fabriciidae Rioja, 1923 (Annelida, Sabellida) reported from the Iberian Peninsula, Balearic and Chafarinas Islands

A taxonomic checklist of the species belonging to the families Sabellidae and Fabriciidae reported from the Iberian Peninsula and the Chafarinas and Balearic Islands is presented. From a thorough revision of the bibliography dealing directly or indirenctly with these groups, it was found that 54 valid species pertaining to 23 genera of Sabellidae and nine species belonging to seven genera of Fabriciidae have been reported to date from the considered area. In this checklist, for each species we provide all the records from the area and all the synonyms used, together with information about their regional geographic distribution.

Keywords: Sabellidae; Fabriciidae; Polychaeta; Iberian Fauna; Checklist.

Recibido/Received: 25/07/2017; Aceptado/Accepted: 26/10/2017; Publicado en línea/Published online: 01/12/2017

Cómo citar este artículo/Citation: López, E. \& Cepeda, D. 2017. Catálogo de las especies de Sabellidae Latreille, 1825 y Fabriciidae Rioja, 1923 (Annelida; Sabellida) citadas en la Península lbérica e islas Baleares y Chafarinas. Graellsia, 73(2): e064. http://dx.doi.org/10.3989/graellsia.2017.v73.184

Copyright: (C) 2017 SAM y CSIC. This is an open-access article distributed under the terms of the Creative Commons Attribution (CC-by) Spain 3.0 License.

\section{Introducción}

Junto con la familia Serpulidae Rafinesque, 1815, las familias Sabellidae Latreille, 1825 y Fabriciidae Rioja, 1923 forman un claro grupo monofilético dentro del orden Sabellida, caracterizado por la existencia de un penacho branquial complejo que rodea la boca (Kupriyanova \& Rouse, 2008) y está formado por dos unidades o lóbulos situados a ambos lados del extremo anterior del peristomio. En cada lóbulo 
se insertan una serie de estructuras longitudinales llamadas radiolos, que tienen prolongaciones laterales, las pínulas (Fig. 1A, B), cuya función es tanto atrapar partículas alimenticias en suspensión como llevar a cabo parte del intercambio gaseoso. Además del penacho branquial, la boca de sabélidos y fabrícidos está rodeada de una serie de estructuras, entre las que destacan unas proyecciones de longitud variable llamadas labios. En sabélidos hay dos pares de labios, dorsal y ventral (Fig. 1D), pero en fabrícidos únicamente existe el par dorsal (Fitzhugh, 1989).

Sabélidos y fabrícidos presentan tanto sedas capilares como uncinos en todos los segmentos setígeros salvo el primero, que presenta exclusivamente sedas capilares. En ambas familias se puede observar una división del metastomio en dos regiones, denominadas tórax y abdomen (Fig. 1A, B). El cambio de región es claramente apreciable por la inversión de la posición de ambos tipos de sedas, ya que mientras que en el tórax las sedas capilares se sitúan en el notopodio y los uncinos en el neuropodio, en el abdomen aparecen en posiciones opuestas (Fig. 1C). Asimismo, se produce un giro en el surco fecal, un canal ciliado que recorre longitudinalmente el cuerpo del animal y se encarga de la remoción de las heces fuera del tubo, de modo que en el tórax aparece en posición dorsal y en el abdomen a nivel ventral. Además, se da la circunstancia de que en muchas especies las sedas presentan diferente aspecto en una y otra región. Las sedas capilares poseen una estructura especial que hizo que en la literatura más antigua se las describiese como limbadas. Sin embargo, los estudios de ultraestructura realizados por Perkins (1984) demostraron que las aparentes expansiones de las sedas son realmente un capuchón subdistal. A partir de esta observación, Fitzhugh (1989) propuso una clasificación de los capilares que es la más utilizada en la actualidad. Según esta, y en términos generales, las sedas capilares pueden tener capuchón ancho, más grueso y extendiéndose a ambos lados del eje central (Fig. 1E), o capuchón estrecho, más fino $\mathrm{y}$, generalmente, extendiéndose a un único lado del eje central (Fig. 1F). Los casos en los que el capuchón es ancho, pero muy corto, y se extiende únicamente a un lado del eje, son definidos como sedas espiniformes (Fig. 1G). También existen otros tipos de sedas capilares con morfologías específicas, siendo el caso de las sedas espatuladas (Fig. $1 \mathrm{H}$ ) o en bayoneta (Fig. 1I) de ciertos géneros de sabélidos o las sedas pseudoespatuladas de los fabrícidos (Fig. 1J).

Los uncinos torácicos de los sabélidos pueden ser de dos tipos: aciculares, con un mango muy alargado que puede ser casi recto o curvarse en su región subdistal (Fig. 1K), o aviculares, con el mango mucho más corto o casi inexistente y una curva subdistal muy pronunciada, denominada pecho (Fig. 1L). En fabrícidos únicamente se encuentran uncinos torácicos de tipo acicular. En el abdomen de los sabélidos pueden aparecer uncinos aviculares similares a los anteriormente
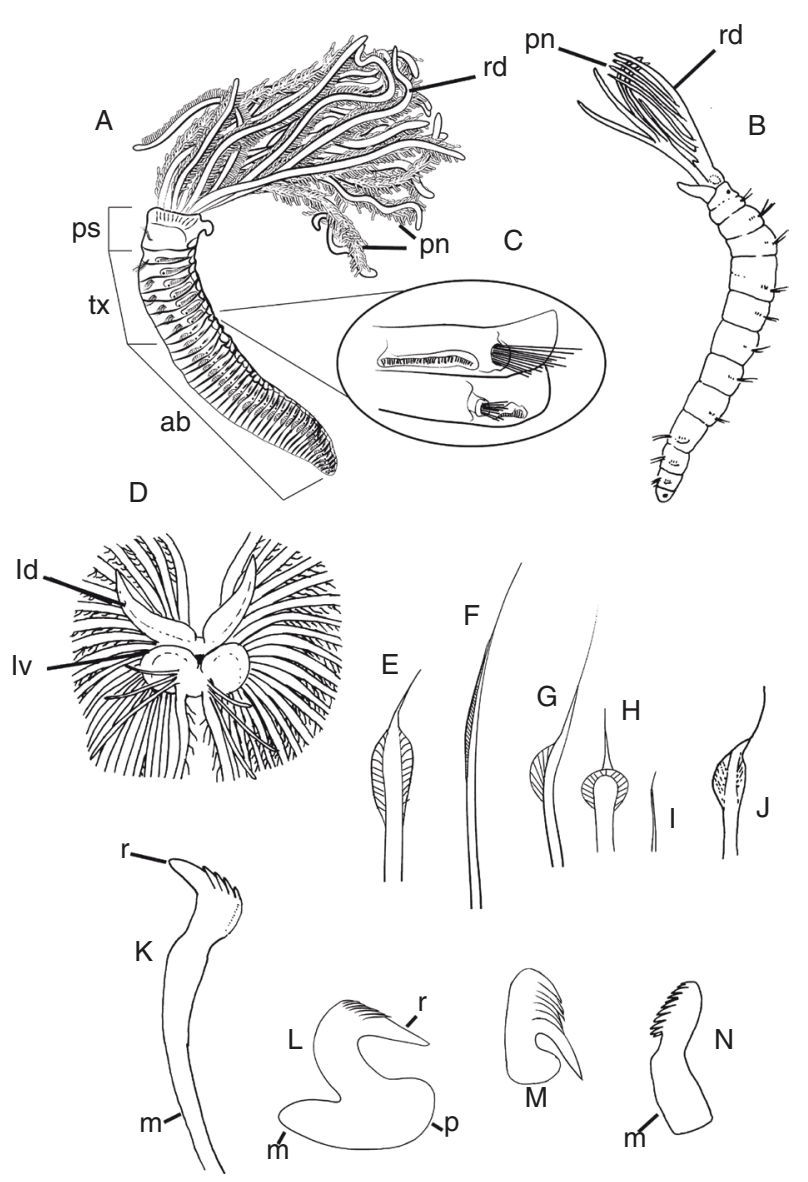

Fig. 1.- Caracteres generales de Sabellidae y Fabriciidae. (A) Sabellidae generalizado, vista lateral del cuerpo; (B) Fabriciidae generalizado, vista lateral del cuerpo; (C) detalle de la región de transición entre el tórax y el abdomen; (D) detalle de la región oral y de la parte interna de la base del penacho, en vista cenital; (E) seda capilar de capuchón ancho; (F) seda capilar de capuchón estrecho; (G) seda capilar espiniforme; $(H)$ seda espatulada; (I) seda en bayoneta; (J) seda pseudoespatulada; (K) uncino torácico acicular; (L) uncino torácico avicular; $(M)$ uncino abdominal en raspador de Sabellidae; (N) uncino abdominal especial de Fabriciidae. Abreviaturas. - ab: abdomen; Id: labio dorsal; Iv: labio ventral; m: mango; p: pecho; pn: pínula; ps: peristomio; r: rostro; rd: radiolo; tx: tórax.

Fig. 1.- General features of de Sabellidae and Fabriciidae. (A) Generalized Sabellidae, lateral view of the body; (B) Fabriciidae generalizado, lateral view of the body; (C) detail of the transitionale región between thorax and abdomen; (D) detail of the mouth region and the internal área of the branchial crown, top view; (E) broadly hooded capillary chaeta; (F) narrowly hooded capillary chaeta; $(G)$ spine-like capillary chaeta; $(H)$ spatulate chaeta; (I) bayonet-like chaeta; $(J)$ pseudospatulate chaeta; (K) acicular thoracic uncinus; (L) avicular thoracic uncinus; (M) rasp-shaped abdominal uncinus of Sabellidae; (N) special abdominal uncinus of Fabriciidae. Abbreviations.ab: abdomen; Id: dorsal lip; Iv: ventral lip; m: handle; p: breast; pn: pinnule; ps: peristomium; r: rostrum; rd: radiole tx: thorax.

descritos o uncinos en raspador (Fig. 1M), que consisten en placas cuadrangulares comprimidas lateralmente que en uno de sus márgenes presentan una o más filas de dentículos (Fitzhugh, 1989). En fabrícidos es característico un uncino abdominal especial, en 
el cual el pecho se extiende hacia la parte basal, de manera que forma una especie de mango largo y aplanado (Fig. 1N).

Rioja (1923) elaboró la primera gran revisión sistemática de la familia, que quedó dividida en tres subfamilias. En este trabajo, Sabellinae fue caracterizada por la presencia de uncinos aviculares en los segmentos torácicos, en contraposición con los uncinos torácicos de forma acicular. Dentro de las especies provistas de este segundo tipo de uncinos torácicos, Fabriciinae fue erigida para incluir todas aquellas cuyos uncinos abdominales se dispusieran en boceles de pequeño tamaño y posición lateral y en cuyo penacho los radiolos fueran libres. A su vez, Myxicolinae, formada únicamente por el género Myxicola Koch in Renier, 1847, se caracterizaba por tener los uncinos abdominales dispuestos en cinturas completas alrededor del segmento y los radiolos unidos por una membrana a lo largo de prácticamente toda su longitud. Poco después, una nueva evaluación de caracteres hizo que Myxicola se transfiriese a Fabriciinae (Johansson, 1927). Esta división en dos subfamilias diferenciadas básicamente por la forma de los uncinos torácicos se mantuvo hasta los exhaustivos estudios filogenéticos realizados por Fitzhugh $(1989,1991)$ en los que se demostró claramente que la forma acicular de los uncinos era plesiomórfica y que la subfamilia Fabriciinae no podía ser caracterizada por dicho estado. Sin embargo, los mismos trabajos confirmaron que una serie de géneros formaban un grupo monofilético junto con Fabricia Blainville, 1828. Por tanto, se decidió mantener ambas subfamilias, pero realizando una profunda enmienda de sus caracteres diagnósticos, de manera que Sabellinae quedó definida por la existencia de radiolos sostenidos por dos o más filas de células esqueléticas de naturaleza cartilaginosa y por la fusión a nivel dorsal de los dos lóbulos del penacho branquial. A su vez, Fabriciinae, formada por especies que carecen de esqueleto radiolar y cuyos lóbulos branquiales están separados desde sus bases, quedó caracterizada por la pérdida de los labios ventrales y por la presencia de un tipo exclusivo de uncino abdominal, con los dientes dispuestos en varias filas paralelas y el pecho trasformado en un manubrio alargado. Ambos taxones se mantuvieron como subfamilias de Sabellidae durante las siguientes décadas (Rouse \& Pleijel, 2001), aunque algunos análisis ya habían indicado la posibilidad de que Fabriciinae pudiera ser una familia dentro del orden Sabellida, con un grado de diferenciación similar al de Serpulidae (Smith, 1991). Este último punto fue confirmado por el análisis combinado de datos morfológicos y moleculares realizado por Kupriyanova \& Rouse (2008), en el que además de la monofilia de ambas familias se demostraba que Fabriciidae era un grupo hermano Serpulidae y que ambos formaban un clado hermano de Sabellidae, de manera que en la actualidad la aceptación de Sabellidae y Fabriciidae como familias separadas es generalizada.

El catálogo de Ariño (1987), en el que se realizó un compendio de toda la bibliografía referida a anélidos poliquetos ibéricos existente hasta el momento, recogió 32 especies válidas pertenecientes a Sabellidae y 4 a Fabriciidae. La literatura posterior ha aumentado el número de especies ibéricas a 54 de sabélidos y 9 de fabrícidos, de las cuales Amphicorina triangulata López \& Tena, 1999 y Bispira primaoculata Cepeda \& Lattig, 2017 tienen su localidad tipo en algún punto del área considerada.

El objetivo del presente trabajo es ofrecer un catálogo exhaustivo de las citas de especies pertenecientes a las familias mencionadas en todo el ámbito ibero-balear, incluidas las islas Chafarinas, completando el catálogo publicado por Alcázar y San Martín (2016) para la familia Serpulidae.

\section{Material y métodos}

Los datos presentados en este trabajo se basan en la búsqueda de citas para la península Ibérica de especies de las familias Sabellidae y Fabriciidae en la bibliografía publicada. Quedan excluidos de esta consideración trabajos académicos no accesibles al público (Tesis de Licenciatura, Proyectos de Fin de Carrera, Grado o Máster o cualquier otro documento asimilable), así como los informes técnicos o de impacto ambiental (de difícil o imposible acceso público), las actas de congresos que no hayan sido publicadas en revistas y las guías de campo o cualquier otra publicación divulgativa, cuyos datos generalmente no han sido evaluados por pares. Se han tenido en cuenta las Tesis Doctorales, ya que las universidades donde son defendidas acostumbran a conservar un ejemplar en sus bibliotecas y permiten el acceso a las mismas con fines de investigación y por su naturaleza son trabajos evaluados por un tribunal de expertos en la materia.

Para cada especie reportada, se da el nombre actualmente aceptado contrastándolo con la base de datos World Register of Marine Species (Costello et al., 2013). En algunos casos que esta necesita actualización, como sucede con varias especies que dicha base incluye en el género Oriopsis Caullery \& Mesnil, 1896 pese a que en la misma este nombre genérico se considera un sinónimo posterior de Amphicorina Claparède, 1864, se ha optado por el nombre genérico válido. Tras el nombre de la especie, se recogen todas sus citas para el ámbito geográfico considerado, indicando el nombre con el que se han publicado. En primer lugar se recogen las citas en las que se usan los nombres genérico y específico válidos en la actualidad, después aquellas citas en las que la especie ha sido incluida en otro género y finalmente las sinonimias; dentro de cada grupo las citas se organizan cronológicamente. Finalmente, se aporta un listado de las localidades en las que la especie ha sido colectada. 


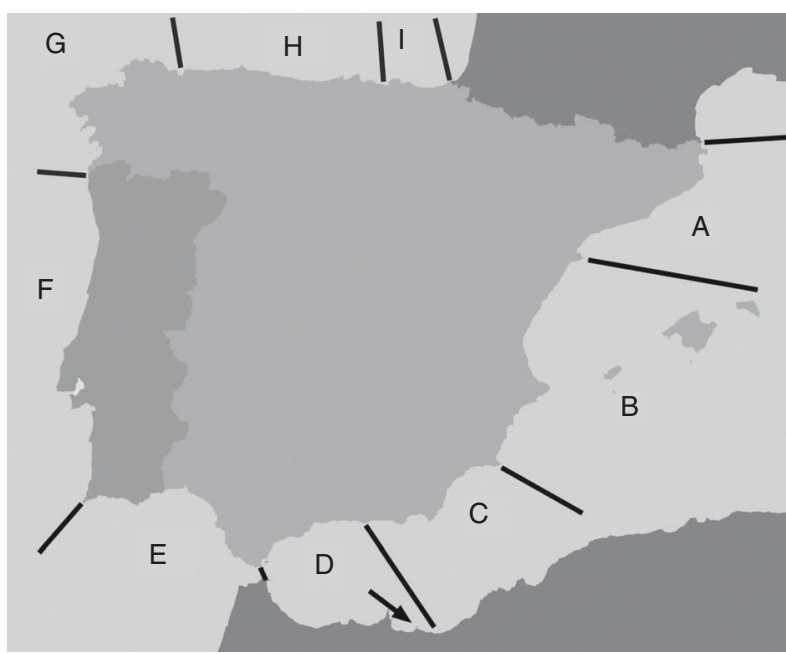

Fig. 2.- Mapa de la zona de estudio mostrando las divisiones biogeográficas propuestas por El Haddad et al. (2013). (A) Cataluña; (B) costa levantina e islas Baleares; (C) Murcia y Almería; (D) mar de Alborán, incluyendo las islas Chafarinas (indicadas por la flecha); (E) golfo de Cádiz; (F) Portugal; (G) Galicia; (H) mar Cantábrico; (I) golfo de Vizcaya.

Fig. 2.- Map of the study area showing the biogeographyc divsions proporsed by El Haddad et al. (2013). (A) Catalonia; (B) Levantine coast and Balearic Islands; (C) Murcia and Almería; (D) Alborán Sea, including Chafarinas Islands (indicated with an arrow); (E) Gulf of Cadiz; (F) Portugal; (G) Galicia; (H) Cantabrian Sea; (I) Bay of Biscay.

Las localidades se han asignado a las diferentes áreas geográficas a partir del criterio propuesto por El-Haddad et al. (2013), según el cual el litoral ibérico quedaría dividido en los sectores siguientes: (A) Cataluña, desde el cabo de Creus hasta el delta del Ebro; (B) costa levantina, desde el delta del Ebro hasta el cabo de Palos, e islas Baleares; (C) Murcia y Almería, desde el cabo de Palos hasta Motril; (D) mar de Alborán, desde Motril hasta el estrecho de Gibraltar, incluyendo las islas Chafarinas; (E) golfo de Cádiz, desde el estrecho de Gibraltar hasta el cabo de San Vicente; (F) Portugal, desde el cabo de San Vicente hasta la desembocadura del Miño; (G) Galicia, desde la desembocadura del Miño hasta la del Eo; $(\mathrm{H})$ mar Cantábrico, desde la desembocadura del Eo hasta Castro-Urdiales; (I) golfo de Vizcaya, desde CastroUrdiales hasta la desembocadura del Bidasoa (Fig. 2).

\section{Resultados}

Familia SABELLIDAE Latreille, 1825

Género Acromegalomma Gil y Nishi, 2017

Acromegalomma lanigerum (Grube, 1846)

Gil (2011): 818-819; Cepeda \& Lattig, 2017: 14-17, fig. 9.

Branchiomma vesiculosum (non Montagu, 1815).- Rioja (1916): 466; (1917a): 65-66; (1917b): 182; (1923): 31-34, figs. 29-36; (1925): 55; (1931): 356-358, Lam. 114, figs. 1-8.
Distribución ibérica.- B: Baleares (Cepeda y Lattig, 2017). D: Málaga (Rioja, 1917b; Cepeda y Lattig, 2017). G: Islas Sisargas y cabo Prior (Cepeda y Lattig, 2017). H: Cantabria (Rioja, 1916, 1917a, 1923, 1925, 1931; Cepeda \& Lattig, 2017).

Acromegalomma vesiculosum (Montagu, 1815)

Campoy (1982): 667; San Martín \& Viéitez (1984): 157, tab. 1; Sardà (1984): 696-697, figs. a-h; (1986a): 73, tab. 1; Ariño (1987): 473-474; Alós (1988): 620-621; Sardà (1991): 347, tab. 1; Rallo et al. (1993): 74, tab. 2; García-Arberas \& Rallo (1994): 74; Méndez \& Cardell (1996): 140, tab. 1; Tena (1996): 427-429; Redondo \& San Martín (1997): 227, tab. 1; Moreira \& Troncoso (2007): 111; Gil (2011): 819; Quintas et al. (2012): 120.

Branchiomma vesiculosum (Montagu, 1815).- Dexter (1992): 80, tab. 1; Cacabelos (2005): 140, 582, 595; Cacabelos et al. (2009): 109.

Megalomma vesiculosum (Montagu, 1813) (sic).- San Martín et al. (1990): 57, tab.1; San Martín \& Aguirre (1991): 161, tab. 1; Moreira (2003): 577; Quintas (2005): 451.

Distribución ibérica.- A: Malgrat de Mar (Campoy, 1982) y Cap de Creus (Alós, 1988), Gerona. Costa de Barcelona (Ariño, 1987; Méndez \& Cardell, 1996). B: Baleares (Sardà, 1991). Costa valenciana (Redondo \& San Martín, 1997). C: Cabo de Palos, Murcia (San Martín \& Viéitez, 1984). Cabo de Gata, Almería (San Martín et al., 1990). D: Algeciras, Cádiz (Sardà, 1984). Nerja, Málaga (San Martín \& Aguirre, 1991). Islas Chafarinas (Tena, 1996). F: Setubal, Estremadura (Rioja, 1923; 1931). Desembocadura del río Sado, Alentejo (Dexter, 1992). G: Baiona (Moreira, 2003; Moreira \& Troncoso, 2007), Vigo (Cacabelos, 2005; Cacabelos et al., 2009) y O Grove, Pontevedra (Quintas, 2005; Quintas et al., 2012). I: Cañón de Capbreton (García-Arberas \& Rallo, 1994).

Comentarios.- Este taxón ha sido frecuentemente confundido con $A$. lanigerum en aguas mediterráneas (Giangrande \& Licciano, 2008) y la revisión de parte del material mediterráneo ibérico (Cepeda \& Lattig, 2017) ha corroborado la frecuencia de estos errores de identificación. Posiblemente la distribución de $A$. vesiculosum esté restringida al Atlántico norte (Giangrande et al. 2015), por lo que sería necesario revisar el resto del material del área mediterránea ibero-balear.

Género Amphicorina Claparède, 1864

Amphicorina armandi Claparède, 1864

Gil (2011): 791.

Amphicorina armandi (Claparède, 1864) (sic).- López \& Viéitez (1999): 379, tab. 3.

Oridia armandi (Claparède, 1864).- Rioja (1917a): 73; (1917b): 184; (1919): 25; (1923): 52-54, figs. 77-82; (1925): 55; (1931): 377-379, Lam. 121, figs. 5-10

Oriopsis armandi (Claparède, 1864).- Desbruyères et al. (1972): 361; Campoy \& Jordana (1978): 54; Amoureux \& Calvário (1981): 52, tab. 1; Campoy (1982): 668; San Martín \& 
Alvarado (1982): 231; Alós et al. (1982): 153, tab. 1; San Martín \& Alvarado (1982): 231; San Martín et al. (1982): 180; Sardà (1982): 169; (1984): 718-719, figs. a-c; (1986a): 73, tab. 1; Alós (1983): 26; (1984): 96, tab. 2; Aguirrezabalaga (1984): 120-122; Templado et al. (1986): 100, tab. 3; Ariño (1987): 477-478; Baratech \& San Martín (1987): 42, Tb. III; Martin (1987): 51, tab. 2; Alós (1988): 643-644; Cardell \& Gili (1988): 86, tab. 1; San Martín et al. (1990): 57, tab.1; Laborda et al. (1991): 78, tab. I; Parapar (1991): 705-707; San Martín \& Aguirre (1991): 161, tab.1; Sardà (1991): 347, tab. 1; López (1995): 544-545; Parapar et al. (1996): 137; Tena (1996): 431434; Tena et al. (2000): 65, tab. 5; Templado et al. (2006): 191.

Oriopsis armandi Claparède, 1864 (sic).- Acero \& San Martín (1986): 18.

Distribución ibérica.- A: Costa catalana (Desbruyères et al., 1972; Martin, 1987). Cap de Creus, Gerona (Alós et al., 1982; Alós, 1983, 1984, 1988). Islas Medas, Gerona (Cardell \& Gili, 1988). B: Valencia (Rioja, 1923, 1931). Islas Columbretes (Campoy, 1982; Ariño, 1987) y Baleares (Ariño, 1987; Sardà, 1991; Soler et al., 1997). C: Águilas, Murcia (Campoy \& Jordana, 1978; Campoy, 1982; Ariño, 1987). Cabo de Gata, Almería (Baratech \& San Martín, 1987; San Martín et al., 1990). D: Málaga (Rioja, 1923, 1931; San Martín et al., 1982; Acero \& San Martín, 1986; San Martín \& Aguirre, 1991). Algeciras, Cádiz (Sardà, 1982). Desde Tarifa, Cádiz, hasta Estepona, Málaga (Sardà, 1984). Islas de Alborán (Templado et al., 1986, 2006; Baratech \& San Martín, 1987) y Chafarinas (Tena, 1996; López \& Viéitez, 1999; Tena et al., 2000). E: Desde Tarifa hasta Barbate, Cádiz (Sardà, 1984). Cádiz (Acero \& San Martín, 1986; Baratech \& San Martín, 1987). F: Peniche, Leiria (Amoureux \& Calvário, 1981). G: La Coruña (Rioja, 1923, 1931). Islas Cies y Ons, Pontevedra (Ariño, 1987). Ferrol, A Coruña (Parapar, 1991; Parapar et al., 1996). H: Cantabria (Rioja, 1917a, 1923, 1931). Asturias (Rioja, 1919, 1923, 1931; Laborda et al., 1991). I: Guipúzcoa (Campoy, 1982; Aguirrezabalaga, 1984; Ariño, 1987).

\footnotetext{
Amphicorina eimeri (Langerhans, 1880)

Oriopsis eimeri (Langerhans, 1880).- Alós et al. (1982): 153, tab. 1; Campoy (1982): 668; Campoy \& Alquézar (1982): 132, tab. 1; Alós (1984): 96, tab. 2; (1988): 644-645; Sardà (1984): 716717, figs. a-e; (1986a): 73, tab. 1; Acero \& San Martín (1986): 18; Ariño (1987): 479-480; San Martín et al. (1990): 57, tab.1; Sardà (1991): 347, tab. 1; Soler et al. (1997): 14, tab.2

Oriopsis eimeri eimeri (Langerhans, 1880).- López (1995): 549; Tena (1996): 434-436; Tena et al. (2000): 65, tab. 5.

Amphicorina eimeri eimeri (Langerhans, 1880).- López \& Viéitez (1999): 379, tab. 3
}

Distribución ibérica.- A: Cap de Creus, Gerona (Alós et al., 1982; Alós, 1984, 1988). B: Islas Baleares(Ariño, 1987; Sardà, 1991; Soler et al., 1997). C: Águilas, Murcia (Campoy, 1982; Campoy \& Alquézar, 1982; Ariño, 1987). Cabo de Gata, Almería (San Martín et al., 1990). D: La Hacienda, Cádiz (Sardà, 1984). Málaga (Acero \& San Martín, 1986). Islas Chafarinas
(López, 1995; Tena, 1996; López \& Viéitez, 1999; Tena et al., 2000). G: Islas Ons, Pontevedra (Ariño, 1987).

\author{
Amphicorina pectinata (Banse, 1957) \\ Giangrande et al. (1999): 200, fig. 5; Serrano (2002): 213-214; \\ Serrano \& Preciado (2007): 20, tb. 1. \\ Oriopsis alata pectinata Banse, 1957.- Tena (1996): 430-431; \\ Tena et al. (2000): 65, tab. 5.
}

Distribución ibérica.- D: Nerja, Málaga (Giangrande et al., 1999). Islas Chafarinas, (Tena, 1996; Tena et al., 2000). H: Santander, Cantabria (Serrano 2002; Serrano \& Preciado, 2007).

Comentarios.- Especie cuya localidad tipo se encuentra en Nueva Zelanda y que fue reportada por primera vez en el mar Mediterráneo por Abbiati et al. (1991), donde se considera una especie introducida por muchos autores (e.g. Occhipinti-Ambrogi et al., 2011). No obstante, el estudio del holotipo, así como los comentarios sobre las especie de Giangrande et al. (1999) y López \& Tena (1999), muestran que los individuos identificados como A. pectinata en el Mediterráneo presentan claras diferencias en el número de apéndices radiolares ventrales, de radiolos y de segmentos abdominales y en la forma de los labios dorsales, indicando que probablemente sean especies distintas. Por ello, resulta necesario revisar el material identificado como A. pectinata en el ámbito ibero-balear.

Amphicorina rovignensis Mikac, Giangrande \& Licciano, 2013

Cepeda \& Lattig, 2017: 2-5, fig. 2 A-C.

Distribución ibérica.- B: Islas Baleares (Cepeda \& Lattig, 2017)

Amphicorina triangulata López \& Tena, 1999

López \& Tena, 1999: 330-334, figs. 1-2; López \& Viéitez (1999): 382; Gil (2011): 792; Cepeda \& Lattig, 2017: 2-5, fig. 2D-E.

Oriopsis cf. brevicollaris (non Rouse, 1990).- López (1995): 545548, fig. 65, P1. 1.

Distribución ibérica.- A: Islas Columbretes (Cepeda y Lattig, 2017). D: Islas Chafarinas (López, 1995; López \& Tena, 1999; López \& Viéitez, 1999).

Género Amphiglena Claparède, 1864

Amphiglena mediterranea (Leydig, 1851)

Rioja (1917a): 69; (1917b): 182; (1919): 25; (1920): 30; (1923): 38-41, figs. 49-57; (1925): 55; (1931): 362-366, Lam. 116, figs. 1-9; Saldanha (1974): 328; Camp (1976): 536, tab. 1; Campoy \& Jordana (1978): 54; Amoureux \& Calvário (1981): 52, tab. 1; Alós et al. (1982): 152, tab. 1; Campoy (1982): 666-667; Campoy \& Alquézar (1982): 132, tab. 1; Monteiro-Marques et 
al. (1982): 131, tab. IV, 136, tab. V, 141, tab. VI; San Martín \& Alvarado (1982): 231; San Martín et al. (1982): 180; Sardà (1982): 169; (1984): 694-695, figs. a-f; (1986a): 73, tab. 1; Alós (1983): 26; (1984): 96, tab. 2; (1988): 613-614; Aguirrezabalaga (1984): 121; Capaccioni-Azzati et al. (1990): 97, tab. 1, 99, tab. II; Ariño (1987): 454-455; Baratech \& San Martín (1987): 42, Tb. III; Capaccioni-Azzati (1987): 460 462; Martin (1987): 51, tab. 2; Cardell \& Gili (1988): 86, tab. 1; Laborda et al. (1991): 78, tab. I; San Martín et al. (1990): 57, tab.1; Parapar (1991): 690-692; San Martín \& Aguirre (1991): 161, tab.1; Sardà (1991): 347, tab. 1; Dexter (1992): 80, tab. 1; López (1995): 531-532; Méndez \& Cardell (1996): 140, tab. 1; Parapar et al. (1996): 134-135; (2009): 297, tab. 1; Tena (1996): 402-404; Soler et al. (1997): 14, tab. 2; López \& Viéitez (1999): 379, tab. 3; Tena et al. (2000): 65, tab. 5.; Serrano (2002): 214; Moreira (2003): 577; Moreira et al. (2006): 221, tab. 3; Templado et al. (2006): 191; Moreira \& Troncoso (2007): 111; Serrano \& Preciado (2007): 20, tab. 1; Gil (2011): 793.

Amphiglena mediterranea Claparède, 1864 (sic).- Acero \& San Martín (1986): 18.

Distribución ibérica.- A: Girona (Camp, 1976). Cap de Creus (Alós et al., 1982; Alós, 1983, 1984, 1988), Blanes (Campoy \& Jordana, 1978; Campoy, 1982) e islas Medas (Cardell \& Gili, 1988), Gerona. Delta del Ebro, Tarragona (Capaccioni-Azzati, 1987; Capaccioni-Azzati et al., 1990). Costa catalana (Martin, 1987). Costa de Barcelona (Méndez \& Cardell, 1996). B: Denia, Alicante y Valencia (Rioja, 1920, 1923, 1931). Baleares (Rioja, 1923, 1931; San Martín \& Alvarado, 1982; Sardà, 1991). Columbretes (Campoy, 1982). C: Águilas, Murcia (Campoy \& Jordana, 1978; Campoy, 1982; Campoy \& Alquézar, 1982). Cabo de Gata, Almería (Baratech \& San Martín, 1987; San Martín et al., 1990). D: Málaga (Rioja, 1917b, 1923, 1931; San Martín et al., 1982; Acero \& San Martín, 1986; San Martín \& Aguirre, 1991). Algeciras, Cádiz (Sardà, 1982). Estepona, Málaga (Sardà, 1984). Islas Chafarinas (López, 1995; Tena, 1996; López \& Viéitez, 1999; Tena et al., 2000) y de Alborán (Templado et al., 2006). E: Cádiz (Sardà, 1984; Acero \& San Martín, 1986). Plataforma continental del Algarve (Dexter, 1992). F: Arrabida (Saldanha, 1974). Peniche, Leiria (Amoureux \& Calvário, 1981, Monteiro-Marques et al., 1982). G: La Coruña (Rioja, 1923, 1931). Ferrol, A Coruña (Parapar, 1991; Parapar et al., 1996, 2009). Baiona, Pontevedra (Moreira, 2003; Moreira et al., 2006; Moreira \& Troncoso, 2007). H: Cantabria (Rioja, 1917a, 1923, 1925, 1931; Serrano, 2002; Serrano \& Preciado, 2007). Asturias (Rioja, 1919, 1923, 1931; Laborda et al., 1991). I: Guipúzcoa (Campoy, 1982; Aguirrezabalaga, 1984).

Género Bispira Krøyer, 1856

Bispira crassicornis (Sars, 1851)

Sabella crassicornis Sars, 1851.- Hartmann-Schröder (1977): 96-97; Ariño (1987): 487; Templado et al. (2002): 194.

Distribución ibérica.- B: Islas Columbretes (Templado et al., 2002). F: Portugal (Hartmann-Schröder, 1977).
Comentarios.- Esta especie tiene como localidad tipo Tromsö (Noruega), aunque también ha sido citada en el mar Mediterráneo por Lo Bianco (1893) y en la costa atlántica de la península ibérica por HartmannSchröder (1977). No obstante, Knight-Jones \& Perkins (1998) observaron importantes diferencias morfológicas entre el material tipo de $B$. crassicornis y los ejemplares descritos en estos dos últimos trabajos, que podrían ser ejemplares jóvenes de Bispira viola (Grube, 1863) o bien pertenecer a una especie aún no descrita del género. Por ello se hace necesaria la revisión de este material y del citado por Templado et al. (2002) para el ámbito ibero-balear.

\section{Bispira fabricii (Krøyer, 1856)}

Gil (2011): 794

Sabella fabricii Krøyer, 1856.- Amoureux (1973): 448; Saldanha (1974): 328; Monteiro-Marques (1979): 194; Campoy (1982): 667; Sardà (1986b): 32; Ariño (1987): 487; Dexter (1992): 80, tab. 1.

Sabella fabrici (Kröger, 1856) (sic).- Templado et al. (1986): 100, tab. 3.

Sabella fabrici Fauvel, 1927 (sic).- Templado et al. (2006): 191.

Distribución ibérica.- A: Blanes, Gerona (Campoy, 1982; Ariño, 1987). Barcelona (Sardà, 1986b). B: Mallorca (Campoy, 1982). Islas Columbretes (Campoy, 1982; Ariño, 1987). D: Isla de Alborán (Templado et al., 1986, 2006). E: Plataforma continental del Algarve (Monteiro-Marques, 1979; Dexter, 1992). F: Arrabida (Saldanha, 1974). I: Costa vasca (Amoureux, 1973).

Comentarios.- La localidad tipo de B. fabricii se encuentra en Groenlandia y según Knight-Jones y Perkins (1998) su distribución biogeográfica esta reducida a aguas del Ártico y el Atlántico norte, con las islas Británicas como límite meridional (Giangrande et al., 2015); por ello es necesario revisar el material reportado de aguas ibéricas, ya que probablemente se trate de una especie distinta.

\section{Bispira melanostigma (Schmarda, 1861)}

Sabella bipunctata Baird, 1865.- Ariño (1987): 486-487; Martin (1987): 51, tab. 2; Méndez \& Cardell (1996): 140, tab. 1;

Distribución ibérica.- A: Costa catalana (Martin, 1987). Costa de Barcelona (Méndez \& Cardell, 1996).

Comentarios.- Bispira melanostigma fue descrita a partir de material colectado en Jamaica y más tarde fue reportada en el mar Mediterráneo por Iroso (1921) como S. bipunctata, un sinónimo posterior de la especie (Hartman, 1959). No obstante, KnightJones \& Perkins (1998) indicaron que el material descrito del Mediterráneo parece corresponderse con ejemplares juveniles de $B$. viola, por lo que sería necesario revisar material de la especie citado en el 
ámbito ibero-balear para determinar si está presente en el área.

Bispira primaoculata Cepeda \& Lattig, 2017

Cepeda \& Lattig, 2017: 6-10, figs. 3-4.

Distribución ibérica.- C: Monte submarino de La Herradura, al suroeste del Cabo Sacratif, Granada (Cepeda \& Lattig, 2017)

Bispira viola (Grube, 1863)

Gràcia et al. (2005): 172-173, fig. 3; Malonda (2008): 84.

Distribución ibérica.- B: Cova des Colls, Mallorca (Gràcia et al., 2005). C: Puerto de Cartagena, Murcia (Malonda, 2008).

\section{Bispira volutacornis (Montagu, 1804)}

Rioja (1917a): 62; (1923): 23-26, figs. 11-20; (1931): 348-350, Lam. 112; Ariño (1987): 455-456; Parapar (1991): 689; Parapar et al. (1996): 135; Gil (2011): 795.

Bispira volutacornis Montagu, 1804 (sic).- Amoureux (1972): 84.

Distribución ibérica.- G: Talud continental de Galicia (Amoureux, 1972). Ferrol, A Coruña (Parapar, 1991; Parapar et al., 1996). H: Cantabria (Rioja, 1917a, 1923, 1931).

Género Branchiomma Kölliker, 1858

Branchiomma bairdi (McIntosh, 1885)

Arias et al., 2013: 164-169. fig. 2; Cepeda \& Rodríguez-Flores (2017): 3-7, figs. 1A-D, 2.

Branchiomma boholense (non Grube, 1878).- Román et al. 2009: 244-248, figs. 2-3; Gil (2011): 797.

Distribución ibérica.- B: Formentera (Cepeda \& Rodríguez-Flores, 2017). C: Mar Menor (Román et al., 2009); puerto de Mazarrón, Murcia (Arias et al., 2013).

Branchiomma bombyx (Dalyell, 1853)

Desbruyères et al. (1972): 357; Amoureux \& Calvário (1981): 52, tab. 1; Sardà (1984): 690-691, figs. a-h; (1986a): 73, tab. 1; Gómez \& San Martín (1985): 115; Ariño (1987): 456-457; Baratech \& San Martín (1987): 42, Tb. III; Martin (1987): 51, tab. 2; Alós (1988): 615-616; San Martín et al. (1990): 57, tab.1; Parapar (1991): 696-698; Sardà (1991): 347, tab. 1; López (1995): 532-533; Parapar et al. (1996): 135; Tena (1996): 405-407; Soler et al. (1997): 14, tab. 2; López \& Viéitez (1999): 382; Serrano (2002): 214-215; Quintas (2005): 451; Serrano \& Preciado (2007): 20, tab. 1; Gil (2011): 797. Quintas et al. (2012): 120; Capa et al. (2013): 641, tab. 1.

Dasychone bombyx (Dalyell, 1853).- Rioja (1917a): 70; (1917b): 182; (1919): 25; (1920): 30; (1923): 43-46, figs. 58-67; (1931): 368-370, Lam. 118, Lam. 120, figs. 4; (1935): 39; Carvalho (1929): 15-16; Saldanha (1974): 328; Camp (1976):
536, tab. 1; Anadón (1981): 111, tab. 1; Alós et al. (1982): 152, tab. 1; Campoy \& Jordana (1978): 54; Campoy (1982): 667; San Martín \& Viéitez (1984): 157, tab. 1.

Dasychone bombix (Dalyell, 1853) (sic).- Aguirrezabalaga (1984): 121.

Distribución ibérica.- A: Costa catalana (Desbruyères et al., 1972; Camp, 1976; Martin, 1987). Cap de Creus (Alós et al., 1982; Alós, 1988) y Blanes (Campoy \& Jordana, 1978; Campoy, 1982; Ariño, 1987), Gerona. B: Valencia (Rioja, 1920, 1923, 1931). Menorca (Rioja, 1923, 1931). Mallorca (Rioja, 1935). Formentera (Ariño, 1987; Soler et al., 1997). Baleares (Sardà, 1991). C: Águilas, Murcia (Campoy, 1982; Ariño, 1987). Cabo de Palos, Murcia (San Martín \& Viéitez, 1984). Cabo de Gata, Almería (Baratech \& San Martín, 1987; San Martín et al., 1990). D: Málaga (Rioja, 1917b, 1923, 1931; Gil, 2011). Islas Chafarinas (López, 1995; Tena, 1996; López \& Viéitez, 1999). E: Tarifa y Zahara de los Atunes, Cádiz (Sardà, 1984). F: Sines, Alentejo (Carvalho, 1929). Arrabida (Saldanha, 1974). Peniche, Leiria (Amoureux \& Calvário, 1981). G: La Coruña (Rioja, 1923, 1931). Vigo (Anadón, 1981). Ferrol, A Coruña (Parapar, 1991; Parapar et al., 1996). O Grove, Pontevedra (Quintas, 2005; Quintas et al., 2012). H: Cantabria (Rioja, 1917a, 1923, 1931; Serrano, 2002; Serrano \& Preciado, 2007). Gijón (Rioja, 1919, 1923, 1931). Asturias (Gómez \& San Martín, 1985). I: Guipúzcoa (Campoy, 1982; Ariño, 1987).

Branchiomma luctuosum (Grube, 1870)

El Haddad et al. (2007): 211, fig. 3; (2008): 2-6, fig. 3; (2012): 277-281; Gil (2011): 798; Capa et al. (2013): 641, tab. 1.

Distribución ibérica.- A: Cataluña (Capa et al., 2013). B: Valencia (El Haddad et al., 2007, 2008). Costa mediterránea entre Benicarló y Santa Pola (El Haddad et al., 2012).

\section{Branchiomma lucullanum (Delle Chiaje, 1828)}

Gil (2011): 798.

Dasychone lucullana (Delle Chiaje, 1828).- Rioja (1917b): 182; (1923): 46-49, figs. 68-72; (1931): 370-372, Lam. 119, Lam. 120, figs. 1-3; Bellan (1960): 28; Amoureux (1972): 85; Camp (1976): 536, tab. 1; Campoy \& Jordana (1978): 54; Alós et al. (1982): 152, tab. 1; Campoy (1982): 667; San Martín et al. (1982): 180; Alós (1983): 26; (1984): 96, tab. 2; Aguirrezabalaga (1984): 120; San Martín \& Viéitez (1984): 157, tab. 1; Acero \& San Martín (1986): 18; Dexter (1992): 80, tab. 1.

Branchiomma lucullana (Delle Chiaje, 1828).- Sardà (1984): 692-693, figs. a-g; (1986a): 73, tab. 1; Ariño (1987): 457-458; Baratech \& San Martín (1987): 42, Tb. III; Alós (1988): 616617; Cardell \& Gili (1988): 86, tab. 1; San Martín et al. (1990): 57, tab.1; Sardà (1991): 347, tab. 1; Parapar (1991): 698-700; López (1995): 533-534; Parapar et al. (1996): 135-136; (2009): 297, tab. 1; López \& Viéitez (1999): 382; Redondo \& San Martín (1997): 227, tab. 1; Tena (1996): 408-409. 
Distribución ibérica.- A: Costa catalana (Camp, 1976). Cap de Creus (Alós et al., 1982; Alós, 1983, 1984, 1988), Blanes (Campoy \& Jordana, 1978; Campoy, 1982; Ariño, 1987) e islas Medas (Cardell \& Gili, 1988), Gerona. B: Costa valenciana (Rioja, 1923, 1931; Redondo \& San Martín, 1997). Menorca (Rioja, 1923, 1931). Baleares (Sardà, 1991). C: Cabo de Palos, Murcia (San Martín \& Viéitez, 1984). Cabo de Gata, Almería (Baratech \& San Martín, 1987; San Martín et al., 1990). D: Málaga (Rioja, 1917b, 1923, 1931; San Martín et al., 1982; Acero \& San Martín, 1986). Algeciras, Cádiz, y Estepona, Málaga (Sardà, 1984). Islas Chafarinas (López, 1995; Tena, 1996; López \& Viéitez, 1999). E: Plataforma continental frente a Cabo Santa María, Algarve (Bellan, 1960; Dexter, 1992). Tarifa y Barbate, Cádiz (Sardà, 1984). F: Portugal (Gil, 2011). G: La Coruña (Rioja, 1923, 1931). Talud continental de Galicia (Amoureux, 1972). Ferrol, A Coruña (Parapar, 1991; Parapar et al., 1996, 2009). I: Guipúzcoa (Campoy, 1982; Aguirrezabalaga, 1984).

\section{Género Chone Krøyer, 1856}

\section{Chone duneri Malmgren, 1867}

Desbruyères et al. (1972): 357; Camp (1976): 536, tab. 1; Amoureux \& Calvário (1981): 52, tab. 1; Campoy (1982): 667; Aguirrezabalaga (1984): 122; Alós (1984): 96, tab. 2; (1988): 630-631; Sardà (1984): 712-713, figs. a-f; (1986a): 73, tab. 1; (1986b): 32; Ariño (1987): 460; Capaccioni-Azzati (1987): 460-462; Capaccioni-Azzati et al. (1990): 97, tab. 1, 99, tab. II; Laborda et al. (1991): 78, tab. I; San Martín et al. (1990): 57, tab.1; Dexter (1992): 80, tab. 1; Rodríguez \& Viéitez (1992): 331, tab. II; Tena et al. (1993): 18, tab.2; López (1995): 534 535; Méndez \& Cardell (1996): 140, tab. 1; Tena (1996): 414 416; Soler et al. (1997): 14, tab. 2; Martin et al. (2000): 5, tab. I; Serrano (2002): 215-218; Serrano \& Preciado (2007): 20, tb. 1; Torres-Gavilá (2008): 444-446; Gil (2011): 801. Sardà et al. (2014): tab. 6.

Chone duneri (Malmgren, 1867) (sic).- Alós et al. (1982): 152, tab. 1

Distribución ibérica.- A: Costa catalana (Desbruyères et al., 1972; Camp, 1976; Méndez \& Cardell, 1996; Sardà et al., 2014). Cap de Creus, Gerona (Alós et al., 1982; Alós, 1984, 1988). Delta del Ebro, Tarragona (Capaccioni-Azzati, 1987; Capaccioni-Azzati et al., 1990). B: Costas valenciana (Tena et al., 1993). Formentera (Soler et al., 1997). C: Cabo de Gata, Almería (San Martín et al., 1990). D: La Línea, Cádiz (Sardà, 1984). Islas Chafarinas (Tena, 1996; TorresGavilá, 2008). E: Punta Umbría, Huelva (Rodríguez \& Viéitez, 1992). F: Peniche, Leiria (Amoureux \& Calvário, 1981). Arrabida (Dexter, 1992). H: Asturias (Laborda et al., 1991). Santander, Cantabria (Serrano \& Preciado, 2007). I: Guipúzcoa (Campoy, 1982; Aguirrezabalaga, 1984).

Comentarios.- El examen de gran parte del material ibero-balear identificado por diversos autores como
C. duneri finalmente demostró que se trataba de individuos incorrectamente identificados y pertenecientes a otras especies de sabélidos (Cepeda \& Lattig, 2017) como Dialychone dunerificta (Tovar-Hernández, Licciano \& Giangrande, 2007) y Euchone cf. pseudolimnicola Giangrande y Licciano, 2006. Cochrane (2000) y Tovar-Hernández et al. (2007) examinaron también material colectado en numerosas localidades del Mediterráneo, el Atlántico centro-oriental y el mar Caribe e identificado como $C$. duneri y encontraron también numerosos errores de identificación. Chone duneri parece presentar una distribución restringida al Ártico y el Atlántico norte (Tovar-Hernández et al. 2007; Giangrande et al. 2015), aunque sería necesario examinar el material colectado en la costa mediterránea del ámbito ibero-balear para confirmar o descartar su presencia en este área.

\section{Chone infundibuliformis Krøyer, 1856}

Rioja (1925): 55-57, figs. 24, 25; (1931): 382-383, Lam. 122; Amoureux \& Calvário (1981): 52, tab. 1; Campoy (1982): 668; Alós (1984): 96, tab. 2; (1988): 633; Sardà (1984): 710-711, figs. a-g; (1986a): 73, tab. 1; Ariño (1987): 461-462; Dexter (1992): 80, tab. 1; Sardà (1991): 347, tab. 1; Pinedo et al. (1999): 67, tab. III; Sardà et al. (2000): 318, Tab II; Templado et al. (2002): 193; Torres-Gavilá (2008): 449-451; Gil (2011): 802-803.

Distribución ibérica.- A: Cap de Creus (Alós, 1988) y Blanes (Pinedo et al., 1999; Sardà et al., 2000), Gerona. B: Baleares (Sardà, 1991). Islas Columbretes (Campoy, 1982; Templado et al., 2002). D: La Hacienda, Cádiz (Sardà, 1984). Islas Chafarinas (Torres-Gavilá, 2008). E: Barbate, Cádiz (Sardà, 1984). F: Peniche, Leiria (Amoureux \& Calvário, 1981). Sines, Alentejo, y Arrábida (Dexter, 1992) H: San Vicente de la Barquera, Cantabria (Rioja, 1925).

Comentarios.- El examen por el segundo autor de este trabajo de material identificado como C. infundibuliformis colectado en Almería (San Martín et al., 1990), la costa asturiana (Gómez \& San Martín, 1985) y la costa gallega (Parapar, 1991; Parapar et al., 1996) ha demostrado que está consituido por ejemplares de Dialychone collaris (Langerhans, 1880) y D. longiseta (Giangrande, 1992). Asimismo, las descripciones de la especie dadas por Rioja (1925) para San Vicente de la Barquera parecen corresponder a otro taxón, ya que difiere por la coloración general del cuerpo, la morfología de los segmentos abdominales y la longitud del mucrón de las sedas torácicas paleadas. La especie parece poseer una distribución restringida al Ártico y el Atlántico norte, con su límite más meridional en las islas Británicas (Giangrande et al., 2015), aunque sería necesario examinar el resto del material recogido en el ámbito ibero-balear para confirmar que la especie no se encuentra presente en el mismo. 
Género Desdemona Banse, 1957

Desdemona ornata Banse, 1957

Ceberio et al. (1998): 38-40, figs. 2-3; Marquiegui \& Aguirrezabalaga (2009): 600; Zorita et al. (2009): 23-26, 53-54, Tabs 4, 5; Carvalho et al. (2011): 104, tab. 3; Gil (2011): 807; Chainho et al., 2015: 203, tab. 1.

Distribución ibérica.- E: Algarve (Chainho et al., 2015). F: Lagoa de Obidos, Leiria (Carvalho et al., 2011). I: Guipúzcoa (Ceberio et al., 1998; Marquiegui \& Aguirrezabalaga, 2009; Zorita et al., 2009).

Género Dialychone Claparède, 1870

Dialychone acustica Claparède, 1870

Desbruyères et al. (1972): 358; Campoy (1982): 668; Ariño (1987): 463; Laborda et al. (1991): 78, tab. I; Torres-Gavilá (2008): 439-440.

Chone acustica (Claparède, 1870).- Serrano et al. (2011): 8, tab. II.

Distribución ibérica.- A: Costa catalana (Desbruyères et al., 1972). Barcelona (Serrano et al., 2011). D: Islas Chafarinas (Torres-Gavilá, 2008). H: Asturias (Laborda et al., 1991). I: Guipúzcoa (Campoy, 1982; Ariño, 1987).

Dialychone arenicola (Langerhans, 1880)

Chone arenicola Langerhans, 1880.- Redondo \& San Martín (1997): 227, 230, fig. 2C-D, tab. 1; Malonda (2008): 84.

Distribución ibérica.- B: Costa valenciana (Redondo \& San Martín, 1997). C: Puerto de Cartagena, Murcia (Malonda, 2008).

\section{Dialychone collaris (Langerhans, 1880)}

Chone collaris Langerhans, 1880.- Camp (1976): 536, tab. 1; Amoureux \& Calvário (1981): 52, tab. 1; Alós et al. (1982): 152, tab. 1; Campoy (1982): 667; Sardà (1982): 169; (1984): 708-709, figs. a-g; (1986a): 73, tab. 1; Alós (1984): 96, tab. 2; (1988): 629-630; San Martín \& Viéitez (1984): 157, tab. 1; Acero \& San Martín (1986): 18; Ariño (1987): 459; Baratech \& San Martín (1987): 42, Tb. III; Capaccioni-Azzati (1987): 462464; Martin (1987): 51, tab. 2; Capaccioni-Azzati et al. (1990): 97, tab. 1, 99, tab. II; San Martín et al. (1990): 57, tab.1; San Martín \& Aguirre (1991): 161, tab.1; Sardà (1991): 347, tab. 1; Dexter (1992): 80, tab. 1; Pardal et al. (1993): 500, 502; Méndez \& Cardell (1996): 140, tab. 1; Tena (1996): 411-414; Soler et al. (1997): 14, tab. 2; Tena et al. (2000): 65, tab. 5; Serrano (2002): 215; Templado et al. (2006): 191; Serrano \& Preciado (2007): 20, tb. 1; Malonda (2008): 85; Torres-Gavilá (2008): 441-443; Gil (2011): 801.

Chone collaris (Langerhans, 1880) (sic).- Templado et al. (1986): 100, tab. 3.

Chone infundibuliformis (non Krøyer, 1856).- Gómez \& San Martín (1985): 115; San Martín et al. (1990): 57, tab.1; Parapar (1991): 707-708; Parapar et al. (1996): 136.

Distribución ibérica.- A: Blanes (Camp, 1976) y Cap de Creus (Alós et al., 1982; Alós, 1984, 1988),
Gerona. Delta del Ebro, Tarragona (CapaccioniAzzati, 1987; Capaccioni-Azzati et al., 1990). Costa catalana (Martin, 1987). Costa de Barcelona (Méndez \& Cardell, 1996). B: Islas Columbretes (Campoy, 1982). Baleares (Sardà, 1991). C: Cabo de Palos, Murcia (San Martín \& Viéitez, 1984). Cabo de Gata, Almería (Baratech \& San Martín, 1987; San Martín et al., 1990). Puerto de Cartagena, Murcia (Malonda, 2008). D: Algeciras, Cádiz (Sardà, 1982, 1984). Málaga (Acero \& San Martín, 1986; San Martín \& Aguirre, 1991). Mar de Alborán (Baratech \& San Martín, 1987; Templado et al., 1986, 2006). Islas Chafarinas (Tena, 1996; Tena et al., 2000; TorresGavilá, 2008). E: Cádiz (Acero \& San Martín, 1986; Baratech \& San Martín, 1987). Plataforma continental del Algarve (Dexter, 1992). F: Peniche, Leiria (Amoureux \& Calvário, 1981). Estuario de Mondego, Coimbra (Pardal et al., 1993). G: Ferrol y Betanzos, A Coruña (Parapar, 1991; Parapar et al., 1996). H: Asturias (Gómez \& San Martín, 1985). Santander, Cantabria (Serrano, 2002; Serrano \& Preciado, 2007).

Dialychone dunerificta (Tovar-Hernández, Licciano \& Giangrande, 2007)

Cepeda \& Lattig, 2017: 10-11, fig. 5.

Chone dunerificta Tovar-Hernández, Licciano \& Giangrande, 2007.- Malonda (2008): 85.

Chone duneri (non Malmgren, 1867).- Acero \& San Martín (1986): 2; Redondo \& San Martín (1997): 227, tab. 2; López \& Viéitez (1999): 382.

Distribución ibérica.- B: Costa valenciana (Redondo \& San Martín, 1997; Cepeda \& Lattig, 2017) C: Puerto de Cartagena, Murcia (Malonda, 2008). D: Torrox, Málaga; islas de Alborán y Chafarinas (López \& Viéitez, 1999; Cepeda \& Lattig, 2017). E: SanctiPetri, Cádiz (Acero \& San Martín, 1986; Cepeda \& Lattig, 2017). G: Islas Sisargas y Cabo Prior, La Coruña (Cepeda \& Lattig, 2017). H: Cabo de Peñas (Cepeda y Lattig, 2017). I: San Sebastián (Cepeda \& Lattig, 2017).

Dialychone longiseta (Giangrande, 1992)

Chone longiseta Giangrande, 1992.- Redondo \& San Martín (1997): 227, 230-232, fig. 2E-F, tab. 1; Zorita et al. (2009): 17, tab. 4.

Distribución ibérica.- B: Costa valenciana (Redondo \& San Martín, 1997). I: Costa vasca (Zorita et al., 2009).

Dialychone usticensis (Giangrande, Licciano \& Castriota, 2006)

Cepeda \& Lattig, 2017: 11-13, fig. 6.

Chone usticensis Giangrande, Licciano \& Castriota, 2006.Malonda (2008): 85. 
Chone filicaudata (non Southern, 1914).- López (1995): 535; López \& Viéitez (1999): 382.

Distribución ibérica.- B: Playa de Corinto, Valencia (Cepeda \& Lattig, 2017) C: Puerto de Cartagena, Murcia (Malonda, 2008). D: Islas Chafarinas (López, 1995; López \& Viéitez, 1999; Cepeda \& Lattig, 2017).

Género Euchone Malmgren, 1866

Euchone analis (Krøyer, 1856)

Amoureux 1987: 76.

Distribución ibérica.- H: Asturias (Amoureux, 1987).

Euchone incolor Hartman, 1965

Martínez \& Adarraga (2001): 145-147, fig. 7; Gil (2011): 809.

Euchone iricolor Hartman, 1965 (sic).- Amoureux (1987): 76.

Distribución ibérica.- F: Aveiro (Amoureux, 1987). I: Guipúzcoa (Martínez \& Adarraga, 2001).

Euchone cf. limnicola Reish, 1959

Euchone cf. limnicola.- Cepeda \& Lattig, 2017: 13-14, fig. 7

Distribución ibérica.- I: Ondárroa y San Sebastián (Cepeda \& Lattig, 2017)

Comentarios.- Euchone limnicola fue descrita a partir de material colectado en California (Reish, 1959) y posteriormente se ha citado como especie alóctona en medios portuarios contaminados de Reino Unido (Cochrane, 2000) y Francia (Guyonnet $\&$ Borg, 2015). Aunque morfológicamente similares, los ejemplares citados en el mar Cantábrico presentan ciertas diferencias con la descripción original de la especie, como son un menor número de radiolos y de segmentos abdominales, así como una depresión prepigidial proporcionalmente más corta (Cepeda \& Lattig, 2016). Esto, junto a su presencia en un medio ambiente completamente distinto (arenas limpias del circalitoral), hizo que estos últimos autores indicasen la posibilidad de encontrarse ante un taxón diferente.

Euchone pseudolimnicola Giangrande \& Licciano, 2006

Gil (2011): 810.

Euchone cf. pseudolimnicola.- Cepeda \& Lattig, 2017: 14-15, fig. 7

Distribución ibérica.- B: Islas Hormigas, Cabo de San Antonio y puerto de Valencia (Cepeda \& Lattig, 2017). C: Murcia (Gil, 2011).
Euchone rosea Langerhans, 1884

Desbruyères et al. (1972): 358; Sardà (1986b): 32; Ariño (1987): 463-464; Dexter (1992): 80, tab. 1; Méndez \& Cardell (1996): 140, tab. 1; Parapar et al. (1996): 136; Redondo \& San Martín (1997): 227, tab. 1; Torres-Gavilá (2008): 451-454, Pl. 8; Gil (2011): 810 .

Distribución ibérica.- A: Costa catalana (Desbruyères et al., 1972). Costa de Barcelona (Sardà 1986b; Méndez \& Cardell, 1996). B: Costa valenciana (Redondo \& San Martín, 1997). D: Islas Chafarinas (Torrés-Gavilá, 2008). F: Portugal (Gil, 2011). G: Ría de Pontevedra (Parapar et al., 1996).

\section{Euchone rubrocincta (Sars, 1862)}

Desbruyères et al. (1972): 358; Campoy (1982): 668; Aguirrezabalaga (1984): 122; Ariño (1987): 464; Redondo \& San Martín (1997): 227, tab. 1; Templado et al. (2002): 193; Gil (2011): 810-811; Martins et al. (2013): 49, tb. 2.

Euchone rubrocincta (Sars, 1861) (sic).- Dexter (1992): 80, tab. 1.

Distribución ibérica.- A: Costa catalana (Desbruyères et al., 1972). B: Costa valenciana (Redondo \& San Martín, 1997). Islas Columbretes (Campoy, 1982; Ariño, 1987; Templado et al., 2002). E: Plataforma continental del Algarve (Dexter, 1992). F: Portugal (Martins et al., 2013). I: Guipúzcoa (Campoy, 1982; Aguirrezabalaga, 1984; Ariño, 1987).

Género Euratella Chamberlin, 1919

Euratella salmacidis (Claparède, 1869)

Euratella salmacilis (Claparède, 1870) (sic).- Ariño (1987) 465.

Laonome salmacidis Claparède (sic).- Desbruyères et al. (1972): 359.

Laonome salmacidis (Claparède, 1870) (sic).- Laborda et al. (1991): 78, tab. I;

Distribución ibérica.- A: Costa catalana (Desbruyères et al., 1972). H: Asturias (Laborda et al., 1991).

\section{Género Hypsicomus Grube, 1870}

Hypsicomus stichophthalmos (Grube, 1863)

Tena (1996): 422-427, Lam. 18, figs. a-f; Pl. 6; Gil (2011): 814.

Hypsicomus stichophthalmus (sic).- Sardà (1991): 347, tab. 1.

Potamilla stichophthalmos (Grube, 1863).- Saldanha (1974): 328

Pseudopotamilla stychophthalmos (Grube, 1863).- Ariño (1987): 486; Martin (1987): 51, tab. 2.

Distribución ibérica.- A: Costa catalana (Martin, 1987). B: Baleares (Sardà, 1991). D: Islas Chafarinas (Tena, 1996). F: Arrabida (Saldanha, 1974).

Género Jasmineira Langerhans, 1880

Jasmineira caudata Langerhans, 1880

Amoureux (1974): 150, fig. 6.5-6.7; Ariño (1987): 470; LópezJamar \& González (1987): 178, tab. 2; Dexter (1992): 80, tab. 
1; Méndez \& Cardell (1996): 140, tab. 1; Parapar et al. (1996): 137; Gil (2011): 815.

Distribución ibérica.- A: Costa de Barcelona (Méndez \& Cardell, 1996). E: Plataforma continental del Algarve (Dexter, 1992). F: Porto Covo, Alentejo (Gil, 2011). G: Plataforma gallega (Amoureux, 1974; López-Jamar \& González, 1987; Parapar et al., 1996).

\section{Jasmineira elegans Saint-Joseph, 1894}

Rioja (1917a): 71; (1923): 55-57, figs. 83-89; (1931): 379380, Lam. 116, figs. 10-16; Desbruyères et al. (1972): 359. Amoureux (1974): 150; Amoureux \& Calvário (1981): 52, tab. 1; Sousa-Reis et al. (1982): 95, tab. 1; Sardà (1982): 169; (1984): 714-715, figs. a-e; (1986a): 73, tab. 1; Alós (1984): 96, tab. 2; (1988): 641-642; Aguirrezabalaga (1984): 122; Ariño (1987): 470-471; Martin (1987): 51, tab. 2; Parapar (1991): 701-703; Sardà (1991): 347, tab. 1; Dexter (1992): 80, tab. 1; Parapar et al. (1992): 120; (1996): 137; López (1995): 542-543; Sola Eslava (1996): 31; López \& Viéitez (1999): 382; Templado et al. (2006): 191; Serrano (2002); 217-218; Cacabelos (2005): 140, 582, 595; Serrano \& Preciado (2007): 20, tb. 1; Lourido et al. (2008): 269, tab. 3; Cacabelos et al. (2009): 109; Lourido (2009): 88; Martins et al. (2013): 49, tab. 2; Gil (2011): 815-816.

Jasmineira elegans (Saint-Joseph, 1894) (sic).- Templado et al. (1986): 100, tab. 3.

Distribución ibérica.- A: Costa catalana (Desbruyères et al., 1972; Martin, 1987). Cap de Creus, Gerona (Alós, 1988). B: Islas Columbretes (Ariño, 1987) y Baleares (Ariño, 1987; Sardà, 1991). D: Algeciras, Cádiz (Sardà, 1982). Desde Tarifa, Cádiz, a Estepona, Málaga (Sardà, 1984). Islas Chafarinas (López, 1995; López \& Viéitez, 1999). Isla de Alborán (Templado et al., 1986, 2006). E: Desde Tarifa hasta Barbate, Cádiz (Sardà, 1984). Plataforma continental del Algarve y ría de Alvor (Dexter, 1992). F: Talud continental de Portugal (Amoureux, 1974). Peniche, Leiria (Amoureux \& Calvário, 1981; Sousa-Reis et al., 1982). Desembocadura del río Sado, Arrabida y Peniche, Leiria (Dexter, 1992). Costas de Alentejo y Algarve (Gil, 2011). Portugal (Mucha \& Costa, 1999; Martins et al., 2013). G: Rías de Ferrol y A Coruña (Parapar, 1991; Parapar et al., 1992, 1996). Rías de Vigo (Cacabelos, 2005; Cacabelos et al., 2009) y de Aldán (Lourido et al., 2008; Lourido, 2009), Pontevedra. H: Cantabria (Rioja, 1917a, 1923, 1931; Serrano, 2002; Serrano \& Preciado, 2007). I: Guipúzcoa (Aguirrezabalaga, 1984; Ariño, 1987; Sola Eslava, 1996).

Género Laonome Malmgren, 1866

Laonome kroyeri Malmgren, 1866 Ariño (1987): 472.

Distribución ibérica.- A: Delta del Ebro (Ariño, 1987).
Género Myxicola Koch in Renier, 1847

Myxicola aesthetica (Claparede, 1870)

Rioja (1917a): 72; (1923): 61-63, figs. 96-99; (1931): 388-390, Lam. 124, figs. 6-7; Sardà (1984): 720-721, figs. a-d; (1986a): 73, tab. 1; Ariño (1987): 475; Alós (1988): 647-648; López (1995): 543; Redondo \& San Martín (1997): 227, tab. 1; Ballesteros (1998): 43; López \& Viéitez (1999): 382; Martin et al. (2000): 5, tab. I; Gil (2011): 820.

Distribución ibérica.- A: Cap de Creus, Gerona (Alós, 1988). Delta del Ebro (Martin et al., 2000). B: Costa valenciana (Redondo \& San Martín, 1997). Islas Baleares (Ballesteros, 1998). D: Estrecho de Gibraltar (Sardà, 1984). Islas Chafarinas (López, 1995; López \& Viéitez, 1999). H: Cantabria (Rioja, 1917a, 1923, 1931).

\section{Myxicola infundibulum (Montagu, 1808)}

Gil (2011): 820.

Myxicola infundibulum (Renier, 1804) (sic).- Rioja (1917a): 71-72; (1917b): 182; (1923): 58-61, figs. 90-95; (1925): 58; (1931): 384-388, Lam. 123, Lam. 124, figs. 1-4, Ariño (1987): 476; Parapar (1991): 708-710; Dexter (1992): 80, tab. 1; Rallo et al. (1993): 75, tab. 2; García-Arberas \& Rallo (1994): 74; Parapar et al. (1996): 134-137; Ballesteros (1998): 43.

Myxicola steenstrupi Krøyer, 1855 (sic).- Rioja (1917b): 182-183; (1920): 30; (1923): 63-64, figs. 100-102; (1931): 390-391, Lam. 124, figs. 9-11.

Myxicola steenstrupi Krøyer, 1856.- Ariño (1987): 477.

Myxicola parasites Quatrefages, 1865.- Rioja (1917b): 183; (1923): 65-66, figs. 103-106; (1931): 391-392, Lam. 124, figs. 12-15.

Distribución ibérica.- B: Valencia (Rioja, 1920, 1923, 1931). Islas Baleares (Ballesteros, 1998). D: Málaga (Rioja, 1917b, 1923). E: Faro, Algarve (Carvalho, 1929). Plataforma continental del Algarve (Dexter, 1992). F: Desembocadura del río Sado, Alentejo (Dexter, 1992). G: Ferrol, A Coruña (Parapar, 1991; Parapar et al., 1996). H: Cantabria (Rioja, 1917a, 1923, 1925, 1931). I: Cañón de Capbreton (Rallo et al., 1993; García-Arberas \& Rallo, 1994).

Género Notaulax Tauber, 1879

Notaulax phaeotaenia (Schmarda, 1861)

Sardà (1991): 347, tab. 1.

Hypsicomus phaeotaenia (Schmarda, 1861).- Campoy (1982): 667; Ariño (1987): 468-469; Templado et al. (2002): 193;

Distribución ibérica.- B: Columbretes (Campoy, 1982; Ariño, 1987; Templado et al., 2002). Baleares (Sardà, 1991).

Género Panousea Rullier \& Amoureux, 1970

Panousea africana Rullier \& Amoureux, 1970

Amoureux (1974): 84-85, fig. 7; Ariño (1987): 480; Gil (2011): 823. 
Distribución ibérica.- F: Talud continental de Portugal (Amoureux, 1974). Sur de Portugal (Gil, 2011).

Género Paradialychone Tovar-Hernández, 2008 Paradialychone filicaudata (Southern, 1914)

Ravara \& Moreira (2013): 538, tab. 1.

Chone filicaudata Southern, 1914.- Desbruyères et al. (1972): 357; Campoy (1982): 667-668; Aguirrezabalaga (1984): 122; Sardà (1986b): 32; Ariño (1987) 461-462; Alós (1988): 632; Dexter (1992): 80, tab. 1; Rodríguez \& Viéitez (1992): 331, tab. II; Parapar et al. (1996): 136; Templado et al. (2002): 193; Torres-Gavilá (2008): 446-448; Gil (2011): 802.

Distribución ibérica.- A: Costa catalana (Desbruyères et al., 1972). Barcelona (Sardà, 1986b). Cap de Creus, Gerona (Alós, 1988). B: Islas Columbretes (Campoy, 1982; Templado et al., 2002). D: Islas Chafarinas (Torres-Gavilá, 2008). E: Plataforma continental del Alentejo (Dexter, 1992). Punta Umbría, Huelva (Rodríguez \& Viéitez, 1992). F: Ría de Aveiro (Ravara \& Moreira, 2013). Arrabida (Dexter, 1992). G: Ría de Pontevedra (Parapar et al., 1996). I: Guipúzcoa (Campoy, 1982; Aguirrezabalaga, 1984).

Género Parasabella Bush, 1905

Parasabella langerhansi (Knight-Jones, 1983)

Demonax langerhansi Knight-Jones, 1983.- López (1995): 539542, fig. 64; Tena (1996): 418-419; Tena et al. (2000): 65, tab. 5; López \& Viéitez (1999): 382.

Distribución ibérica.- D: Islas Chafarinas (López, 1995; Tena, 1996; López \& Viéitez, 1999; Tena et al., 2000).

Parasabella saxicola (Grube, 1861)

Demonax brachychona (Claparède, 1870).- Capaccioni-Azzati (1987): 466-468, Lam. 35, figs. A-F; Capaccioni-Azzati et al. (1992): 118-120, fig. 3; López (1995): 535-539, fig. 63; Tena (1996): 417-418; López \& Viéitez (1999): 382; Gil (2011): 806.

Distribución ibérica.- A: Delta del Ebro, Tarragona (Capaccioni-Azzati, 1987; Capaccioni-Azzati et al., 1992). D: Islas Chafarinas (López, 1995; Tena, 1996; López \& Viéitez, 1999). Punta de la Chullera, Málaga (Gil, 2011).

Comentarios.- Knight-Jones (1983) revisó el material tipo de Sabella saxicola, redescribiéndolo y transfiriendo la especie al género Demonax. Posteriormente, sugirió la sinonimia de $D$. saxicola (Grube, 1861) y D. brachychona (Claparède, 1870), con preferencia del segundo nombre (Knight-Jones et al., 1991). Este criterio fue seguido por Giangrande (1994) y en general por los autores que han estudiado material del Mediterráneo o de la costa atlántica europea. Sin embargo, de acuerdo con el Principio de Prioridad del Código Internacional de Nomenclatura Zoológica
(ICZN, 1999), el nombre específico válido es saxicola (Tovar-Hernández \& Harris, 2010).

Parasabella tenuicollaris (Grube, 1870)

Cepeda \& Lattig, 2017: 17-18, fig. 10.

Distribución ibérica.- B: Islas Columbretes y Baleares (Cepeda \& Lattig, 2017). D: Isla de Alborán (Cepeda \& Lattig, 2017). H: Cabo Peñas, Asturias (Cepeda \& Lattig, 2017).

Parasabella tommasi (Giangrande, 1994)

Cepeda \& Lattig, 2017: 18-19, fig. 11.

Distribución ibérica.- B: Isla Espardell (Cepeda \& Lattig, 2017)

Género Perkinsiana Knight-Jones, 1983

Perkinsiana rubra (Langerhans, 1880)

Serrano (2002): 218; Serrano \& Preciado (2007): 20, tab. 1; Gil (2011): 823-824.

Potamilla rubra Langerhans, 1880.- Rioja (1919): 23-25, fig. 10

Potamilla rubra Rioja, 1918 (sic).- Ariño (1987) 483

Distribución ibérica.- H: Gijón (Rioja, 1919). Cantabria (Rioja, 1919; Serrano, 2002; Serrano \& Preciado, 2007).

Perkinsiana socialis (Langerhans, 1884)

Tena (1996): 439-444, Lam. 20, figs. a-g.

Distribución ibérica.- D: Islas Chafarinas (Tena, 1996).

Género Potamethus Chamberlin, 1919

Potamethus filiformis Hartmann-Schröder, 1977

Hartmann-Schröder, 1977: 97, figs. 70-75; Ariño (1987): 480; Gil (2011): 824-825.

Distribución ibérica.- F: Cabo Sardão, Alentejo (Hartmann-Schröder, 1977).

Género Potamilla Malmgren, 1866

Potamilla torelli Malmgren, 1866

Rioja (1917a): 65; (1917b): 182; (1920): 30; (1923): 29-30, figs. 23-28; (1925): 54; (1931): 353-354, Lam. 113, figs. 3-12; (1935): 39; Camp (1976): 538, tab. 2; Campoy \& Jordana (1978): 54; Amoureux \& Calvário (1981): 52, tab. 1; Campoy (1982): 667; Sardà (1982): 169; (1984): 704-705, figs. a-g; (1986a): 73, tab. 1; Alós (1984): 96, Ta. 2; (1988): 623-624; Aguirrezabalaga (1984): 121; Ariño (1987): 484-485; Martin (1987): 51, tab. 2; San Martín et al. (1990): 57, tab.1; Parapar (1991): 694-696; San Martín \& Aguirre (1991): 161, tab.1; Sardà (1991): 347, tab. 1; Parapar et al. (1996): 138; (2009): 297, tab. 1; Tena (1996): 444-446; Serrano (2002): 219-220; 
Serrano \& Preciado (2007): 20, tab. 1; Martins et al. (2013): 49, tab. 2.

Potamilla torelli Malmgren, 1865 (sic).- Amoureux (1972): 84; (1973): 449; Saldanha (1974): 328

Potamilla torelli (Malmgren, 1867) (sic).- Alós et al. (1982): 152, tab. 1; Alós (1983): 26; Altuna et al. (1983): 140.

Distribución ibérica.- A: Blanes (Camp, 1976; Campoy \& Jordana, 1978; Campoy, 1982) y Cap de Creus (Alós et al., 1982; Alós, 1984, 1988), Gerona. Costa catalana (Ariño, 1987; Martin, 1987). B: Valencia (Rioja, 1920). Baleares (Rioja, 1935; Ariño, 1987; Sardà, 1991). Islas Columbretes (Campoy, 1982; Ariño, 1987). Isla Portichol, Alicante (Campoy, 1982). C: Cabo de Gata, Almería (San Martín et al., 1990). D: Málaga (Rioja, 1917b). Algeciras, Cádiz (Sardà, 1982). Estepona (Sardà, 1984) y Nerja (San Martín \& Aguirre, 1991), Málaga. Islas Chafarinas (Tena, 1996). E: Barbate, Cádiz (Sardà, 1984). F: Peniche, Leiria (Amoureux \& Calvário, 1981). Portugal (Martins et al., 2013). G: Talud continental de Galicia (Amoureux, 1972). Islas Ons, Pontevedra (Ariño, 1987). Ferrol, A Coruña (Parapar, 1991; Parapar et al., 1996, 2009). H: Cantabria (Rioja 1917a, 1925; Serrano, 2002; Serrano \& Preciado, 2007). Asturias (Amoureux, 1973). I: Guipúzcoa (Altuna et al., 1983; Campoy, 1982; Aguirrezabalaga, 1984; Ariño, 1987)

Género Pseudopotamilla Bush, 1905

Pseudopotamilla reniformis (O.F. Müller, 1771)

Potamilla reniformis (Müller, 1771).- Rioja (1916): 465; (1917a): 63-64, fig. 19; (1917b): 182; (1917c): 491; (1920): 30; (1923): 27-29, figs. 21, 22; (1925): 54; (1931): 350-353, Lam. 113, figs. 1-2; Amoureux (1973): 448; Camp (1976): 538, tab. 1; Campoy \& Jordana (1978): 54; Hartmann-Schröder (1979): 89.

Potamilla reniformis (Müller, 1788) (sic).- Bellan (1959): 337; (1960): 27; Amoureux (1974): 148, fig. 6.1-6.4; Saldanha (1974): 328; Alós et al. (1982): 152, tab. 1; Campoy (1982): 667; Campoy \& Alquézar (1982): 132, tab. 1; MonteiroMarques et al. (1982): 136, tab. V, 141, tab. VI; Aguirrezabalaga (1984): 121; Sardà (1984): 702-703, figs. a-i; (1986a): 73, tab. 1; Gómez \& San Martín (1985): 115; Baratech \& San Martín (1987): 42, Tb. III; San Martín \& Aguirre (1991): 161, tab.1; Dexter (1992): 80, tab. 1; Serrano (2002): 220-221.

Potamilla reniformis (Linnaeus, 1788) (sic).- Amoureux \& Calvário (1981): 52, tab. 1; Ariño (1987): 481-482; Parapar et al. (1996): 138; Moreira (2003): 577; Moreira \& Troncoso (2007): 111.

Pseudopotamilla reniformis (Bruguière, 1789) (sic).-CapaccioniAzzati (1987): 471-474; Martin (1987): 51, tab. 2; Alós (1988): 625-626; Sardà (1991): 347, tab. 1; López (1995): 549-550; Redondo \& San Martín (1997): 227, tab. 1; Serrano \& Preciado (2007): 20, tab. 1; Gil (2011): 828.

Distribución ibérica.- A: Cadaqués, L'Ametlla de Mar (Camp, 1976), Malgrat de Mar (Campoy, 1982) y Cap de Creus (Alós et al., 1982; Alós, 1988), Gerona. Costa catalana (Ariño, 1987; Martin, 1987). Delta del Ebro, Tarragona (Capaccioni-Azzati, 1987). B: Costa valenciana (Rioja, 1920; Redondo \& San Martín, 1997). Islas Baleares (Sardà, 1991). C:
Águilas, Murcia (Campoy \& Jordana, 1978; Campoy, 1982; Campoy \& Alquézar, 1982; Ariño, 1987). Cabo de Gata, Almería (Baratech \& San Martín, 1987). D: Málaga (Rioja, 1917b). Plataforma de Alborán (Bellan, 1959). Estepona (Sardà, 1984) y Nerja (San Martín \& Aguirre, 1991), Málaga. Isla de Alborán (Gil, 2011). E: Plataforma continental frente a Cabo Santa María, Algarve (Bellan, 1960). Barbate, Cádiz (Sardà, 1984). F: Talud continental de Portugal (Amoureux, 1974). Costa portuguesa (HartmannSchröder, 1979). Peniche, Leiria (Amoureux \& Calvário, 1981; Monteiro-Marques et al., 1982). Arrabida (Saldanha, 1974). Desembocadura del río Sado, Alentejo (Dexter, 1992). G: Islas Cíes y Ons, Pontevedra (Ariño, 1987). Costa de Galicia (Parapar et al., 1996). Baiona, Pontevedra (Moreira, 2003; Moreira \& Troncoso, 2007). H: Gijón (Rioja, 1916, 1917a, 1917c). Cantabria (Rioja, 1916, 1917a, 1925; Serrano, 2002; Serrano \& Preciado, 2007). Asturias (Amoureux, 1973; Gómez \& San Martín, 1985). I: Costa vasca (Amoureux, 1973). Guipúzcoa (Campoy, 1982; Aguirrezabalaga, 1984; Ariño, 1987)

Comentarios.- La especie ha sido tradicionalmente considerada un sinónimo más antiguo de $P$. saxicava (Quatrefages, 1866), estando en desuso el segundo nombre. Sin embargo, una reciente revisión de especies del género (Knight-Jones et al., 2017) ha demostrado que se trata de dos especies distintas. La principal diferencia entre ellas se observa en el margen dorsal del collar, que en $P$. reniformis tiene forma de $\mathrm{V}$ con prolongaciones dorsales bien desarrolladas que cubren las bases de los lóbulos branquiales y en $P$. saxicava posee forma convexa con prolongaciones dorsales mucho más bajas y de extremos redondeados. Según estos autores, las citas de $P$. reniformis para el ámbito ibero-balear en las que se incluye una descipción de los ejemplares (Rioja, 1916, 1917a, $1917 \mathrm{~b}, 1917 \mathrm{c}, 1925)$ se corresponden realmente con $P$. saxicava, que se encontraría distribuida por las costas atlánticas de Francia y la península Ibérica, el mar Mediterráneo y el mar Rojo, mientras que $P$. reniformis quedaría restringida al Atlántico norte. Por tanto es necesario revisar los ejemplares ibero-baleares reportados como $P$. reniformis para establecer su verdadera identidad específica.

\section{Género Sabella Linnaeus, 1767 \\ Sabella discifera Grube, 1874}

Gil (2011): 829.

Sabella variabilis Langerhans, 1884.- Ariño (1987): 490.

Branchiomma linaresi Rioja, 1917.- Rioja (1917a): 66-69, fig. 20; (1923): 35-38, figs. 37-48; (1931): 360-362, Lam. 114, figs. 9-12, Lam. 115; Camp (1976): 536, tab. 1; Cacabelos (2005): 140, 582, 595; Cacabelos et al. (2009): 109.

Megalomma linaresi (Rioja, 1917).- Campoy (1982): 667; Ariño (1987): 473; Alós (1988): 619-620; Sardà (1991): 347, tab. 1; Templado et al. (2002): 193. 
Distribución ibérica.- A: Cap de Creus, Gerona (Camp, 1976; Alós, 1988). B: Islas Columbretes (Campoy, 1982; Ariño, 1987; Templado et al., 2002). Baleares (Sardà, 1991). G: Vigo, Pontevedra (Cacabelos, 2005; Cacabelos et al., 2009). H: Cantabria (Rioja, 1917a, 1923, 1931).

\section{Sabella pavonina Savigny, 1822}

Carvalho (1929): 15; Bellan (1959): 336-337; Saldanha (1974): 328; Camp (1976): 538, tab. 1; Ariño (1987): 488-489; San Martín et al. (1990): 57, tab.1; Sardà (1991): 347, tab. 1; Dexter (1992): 80, tab. 1; Tena (1996): 447-449; Templado et al. (1993): 105, fig. 85; Ballesteros (1998): 43-44; López \& Viéitez (1999): 382; Martin et al. (2000): 5, tab. I; Serrano (2002): 221; Cacabelos (2005): 140, 582, 595; Torres-Gavilá (2008): 455-457; Lourido et al. (2008): 269, tab. 3; Cacabelos et al. (2009): 109; Lourido (2009): 88; Gil (2011): 829-830.

Sabella pavonina Savigny, 1826 (sic).- Rioja (1917a): 63; (1917b): 181; (1920): 30; (1923): 17-20, figs. 1-3, 5; (1925): 54; (1931): 339-342, Lam. 109, figs. 1-3, Lam. 111, fig. 6; (1935): 38; Monteiro-Marques (1979): 194.

Sabella pavonina Savigny, 1820 (sic).- Amoureux (1972): 84; Campoy (1982): 667.

Sabella pavonina Savigny, 1818 (sic).- Amoureux (1974): 148; López (1995) : 550-551.

Sabella pavonina Saint Joseph, 1894 (sic).- Rallo et al., 1993: 74, tab. 2; García-Arberas \& Rallo (1994): 74.

Sabella penicillus (non Linnaeus, 1767).- Hartmann-Schröder (1977): 97; Campoy \& Jordana, (1978): 54; Sardà (1984): 700-701, figs. a-h; (1986a): 73, tab. 1; Ariño (1987): 489-490; Baratech \& San Martín (1987): 42, Tb. III; Martin (1987): 51, tab. 2; Parapar (1991): 692-694; Parapar et al. (1996): 138139; Moreira (2003): 577; Quintas (2005): 451; Moreira \& Troncoso (2007): 111.

Distribución ibérica.- A: Gerona (Rioja, 1923, 1931; Camp, 1976; Campoy \& Jordana, 1978; Campoy, 1982; Ariño, 1987). Costa catalana (Martin, 1987). Delta del Ebro (Martin et al., 2000). B: Vinaroz, Castellón (Rioja, 1920). Islas Baleares (Rioja, 1923, 1931; Campoy, 1982; Sardà, 1991; Ballesteros, 1998). Valencia (Rioja, 1923, 1931) C: Cabo de Gata, Almería (Baratech \& San Martín, 1987; San Martín et al., 1990; Gil, 2011). D: Málaga (Rioja, 1917b, 1923, 1931). Algeciras, Cádiz (Sardà, 1984). Mar de Alborán (Bellan, 1959; Baratech \& San Martín, 1987; Templado et al., 1993; Gil, 2011). Islas Chafarinas (López, 1995; Tena, 1996; López \& Viéitez, 1999; Torres-Gavilá, 2008). E: Chiclana, Cádiz (Baratech \& San Martín, 1987). Plataforma continental del Algarve (Monteiro-Marques, 1979; Dexter, 1992). F: Vilanova de Milfontes, Alentejo (Carvalho, 1929). Talud (Bellan, 1960; Amoureux, 1974) y plataforma continentales de Portugal (Hartmann-Schröder, 1977). Arrabida (Saldanha, 1974). Rías de Mira y Sado, Alentejo (Dexter, 1992). G: Rías de Vigo (Rioja, 1935; Cacabelos, 2005; Cacabelos et al., 2009), de Arosa (Quintas, 2005) y de Aldán (Lourido et al., 2008; Lourido, 2009), Pontevedra. Talud continental de Galicia (Amoureux, 1972). Islas Cíes, Pontevedra
(Ariño, 1987). Ferrol, A Coruña (Parapar, 1991; Parapar et al., 1996). Baiona, Pontevedra (Moreira, 2003; Moreira \& Troncoso, 2007). H: Cantabria (Rioja, 1917a, 1923, 1925, 1931; Serrano, 2002). I: Cañón de Capbreton (Rallo et al., 1993; GarcíaArberas \& Rallo, 1994).

Sabella spallanzanii (Gmelin en Linneo, 1791)

Martin et al. (2000): 5, tab. I; Gil (2011): 830; Quintas et al. (2012): 120

Spirographis spallanzanii Viviani, 1805 (sic).- Rioja (1917a): 61-62; (1917b): 181; (1923): 20-23, figs. 4, 6-9; (1925): 54; (1931): 344-348, Lam. 110, 111, figs. 1-5; (1935) : 39; Carvalho (1929) : 15; Saldanha (1974): 328; Campoy (1982): 667; Sardà (1982): 169; (1984): figs. a-e; (1986a): 73, tab. 1; San Martín \& Viéitez (1984): 157, tab. 1; Ariño (1987): 490 491; Baratech \& San Martín (1987): 42, Tb. III; Alós (1988): 627-628; San Martín et al. (1990): 58, tab.1; Parapar (1991): 700-701; López (1995): 551-552; Parapar et al. (1996): 139; Bustamante (2013): 88, tab. 1.

Spyrographiis spallanzanii Viviani, 1805 (sic).- Rioja (1920): 30. Spirographiis spallanzani Viviani, 1805 (sic).- Henke (1972): 350; Camp (1976): 538, tab. 1; Aguirrezabalaga (1984): 120.

Spirographis spallanzanii Quatrefages, 1866 (sic).- Nobre (1937): 15.

Sabella spallanzani (Viviani, 1805) (sic).- Sardà (1991): 347, tab. 1; Tena (1996): 449-451.

Sabella spallanzanii (Viviani, 1805).- Serrano (2002): 221; Serrano \& Preciado (2007): 20, tb. 1.

Distribución ibérica.- A: Blanes (Camp, 1976) y Cap de Creus (Alós, 1988), Gerona. Delta del Ebro (Martin et al., 2000). B: Valencia (Rioja, 1920). Baleares (Rioja, 1935; Sardà, 1991). C: Águilas, Murcia (Campoy, 1982). Cabo de Palos, Murcia (San Martín \& Viéitez, 1984). Cabo de Gata, Almería (Baratech \& San Martín, 1987; San Martín et al., 1990). D: Málaga (Rioja, 1917b). Punta de la Mona, Granada (Henke, 1972). Algeciras, Cádiz (Sardà, 1982, 1984). Mar de Alborán (Baratech \& San Martín, 1987). Islas Chafarinas (López, 1995; Tena, 1996). E: Portimão, Algarve (Nobre, 1937). F: Sines y Vilanova de Milfontes, Alentejo (Carvalho, 1929). Arrabida (Saldanha, 1974). G: Ferrol, A Coruña (Parapar, 1991; Parapar et al., 1996). O Grove, Pontevedra (Quintas et al., 2012). H: Cantabria (Rioja, 1917a, 1925; Serrano, 2002; Serrano \& Preciado, 2007). I: Guipúzcoa (Campoy, 1982; Aguirrezabalaga, 1984).

Familia FABRICIIDAE Rioja, 1923

Género Fabricia Blainville, 1828

Fabricia stellaris (O. F. Müller, 1774)

Serrano (2002): 217; Serrano \& Preciado (2007): 20, tab. 1; Gil (2011): 812-813; Quintas et al. (2012): 120.

Fabricia stellaris stellaris (O. F. Müller, 1774).- Moreira (2003): 577; Quintas (2005): 451; Moreira \& Troncoso (2007): 111.

Fabricia sabella (Ehrenberg, 1836).- Rioja (1917b): 183-184; Alós et al. (1982): 152, tab. 1; Acero \& San Martín (1986): 18; Capaccioni-Azzati (1987): 469-471; Tena (1996): 419-422; 
Soler et al. (1997): 14, tab. 2; López \& Viéitez (1999): 379, tab. 3 .

Fabricia sabella (Ehrenberg, 1837) (sic).- Saldanha (1974): 328; Amoureux \& Calvário (1981): 52, tab. 1; Castro \& Viegas (1981): 76, tab. 1; Anadón (1981): 111, tab. 1; Campoy (1982): 668; Campoy \& Alquézar (1982): 132, tab. 1; MonteiroMarques et al. (1982): 131, tab. IV, 136, tab. V, 141, tab. VI; San Martín et al. (1982): 180; Sardà (1982): 169; (1984): 706-707, figs. a-e; (1986a): 73, tab. 1; Alós (1983): 26; (1984): 96, tab. 2; (1988): 635-636; Altuna et al. (1983): 140; Aguirrezabalaga (1984): 121,122; San Martín \& Viéitez (1984): 157, tab. 1; Ariño (1987): 465-467; Martin (1987): 51, tab. 2; Cardell \& Gili (1988): 86, tab. 1; Sardà (1991): 347, tab. 1; Parapar (1991): 703-705; San Martín et al. (1990): 57, tab.1; Junoy \& Viéitez (1990b): 334, tab. 3; (1992): 340, tab. I; San Martín \& Aguirre (1991): 161, tab.1; López (1995): 553-554; Junoy (1996): 37; Cacabelos (2005): 140, 582, 595; Parapar et al. (1996): 136-137; (2009): 297, tab. 1; Bustamante et al. (2007): 401, tab. 1; Cacabelos et al. (2009): 109.

Fabricia sabella (Ehrenberg, 1828) (sic).- San Martín \& Alvarado (1982): 231.

Fabricia fabricii (O.F. Müller) (sic).- Rioja (1923): 50-51, figs. 73-76; (1925): 55; (1931): 376-377, Lam. 121, figs. 1-4.

Novafabricia bilobata Martin \& Giangrande, 1991: 114-120, figs. 2-6; Martin et al. (2000): 5, tab. I.

Distribución ibérica.- A: Malgrat de Mar (Campoy, 1982) y Cap de Creus (Alós et al., 1982; Alós, 1983, 1984, 1988), Gerona. Delta del Ebro, Tarragona (Capaccioni-Azzati, 1987; Martin \& Giangrande, 1991; Martin et al., 2000). Costa catalana (Ariño, 1987; Martin, 1987). Islas Medas, Gerona (Cardell \& Gili, 1988). B: Isla de Portichol, Alicante, e islas Columbretes, Csatellón (Campoy, 1982). Islas Baleares (Ariño, 1987; Sardà, 1991; Soler et al., 1997). C: Águilas (Campoy, 1982; Campoy \& Alquézar, 1982; Ariño, 1987) y cabo de Palos (San Martín \& Viéitez, 1984), Murcia. Cabo de Gata, Almería (San Martín et al., 1990). D: Málaga (Rioja, 1917b, 1923, 1931; San Martín et al., 1982; Sardà, 1984; Acero \& San Martín, 1986; San Martín \& Aguirre, 1991). Algeciras, Cádiz (Sardà, 1982). Islas Chafarinas (López, 1995; Tena, 1996; López \& Viéitez, 1999). E: Cádiz (Sardà, 1984; Acero \& San Martín, 1986). F: Arrabida (Saldanha, 1974). Peniche, Leiria (Amoureux \& Calvário, 1981; Monteiro-Marques et al., 1982). Estuario del Tajo (Castro \& Viegas, 1981). G: Vigo (Anadón, 1981; Cacabelos et al., 2009), islas Ons (Ariño, 1987), Baiona (Moreira, 2003; Moreira \& Troncoso, 2007) y O Grove (Quintas, 2005; Quintas et al., 2012), Pontevedra. Ferrol, A Coruña (Parapar, 1991; Parapar et al., 1996, 2009). Ría de Foz, Lugo (Junoy \& Viéitez, 1990b, 1992; Junoy, 1996). H: Cantabria (Rioja, 1925, 1931; Campoy, 1982; Ariño, 1987; Serrano, 2002; Serrano \& Preciado, 2007). I: Guipúzcoa (Campoy, 1982; Altuna et al., 1983; Aguirrezabalaga, 1984; Ariño, 1987). Ría de Bilbao (Bustamante et al., 2007).

Género Fabriciola Friedrich, 1939

Fabriciola baltica Fiedrich, 1939
Ariño (1987): 467; Alós (1988): 637, fig. 92; San Martín et al. (1990): 58, tab.1; Gil (2011): 813.

Distribución ibérica.- A: Cap de Creus, Gerona (Alós, 1988). C: Cabo de Gata (San Martín et al., 1990).

\section{Fabriciola tonerella Banse, 1959}

Campoy (1982): 668; Campoy \& Alquézar (1982): 132, tab. 1; Aguirrezabalaga (1984): 122, 125; Alós (1984): 96, tab. 2; Ariño (1987): 468; López (1995): 554-558, fig. 66, lam. 2; López \& Viéitez (1999): 379, tab. 3; Bick (2005): 134-142, figs. 1, 2; Gil (2011): 813.

Fabriciola tonerella (Banse, 1956) (sic).- Alós et al. (1982): 153, tab. 1.

Fabriciola cf. baltica non Fiedrich, 1939.- Acero \& San Martín (1986): 19, fig. 10.

Distribución ibérica.- A: Gerona (Alós et al., 1982; Alós, 1984; Bick, 2005). B: Islas Columbretes (Campoy, 1982). Ibiza (Bick, 2005). C: Águilas, Murcia (Campoy, 1982; Campoy \& Alquézar, 1982; Ariño, 1987). D: Málaga (Acero \& San Martín, 1986). Islas Chafarinas (López, 1995; López \& Viéitez, 1999). I: Guipúzcoa (Campoy, 1982; Aguirrezabalaga, 1984; Ariño, 1987)

Género Manayunkia Leidy, 1858

Manayunkia aestuarina (Bourne, 1883)

Ariño (1987): 472; Junoy \& Viéitez (1990a): 86-90, fig. 2; (1990b): 334, tab. 3; (1992): 340, tab. I; Junoy (1996): 37; Parapar et al. (1996): 137; Marquiegui \& Aguirrezabalaga (2009): 600; Gil (2011): 816-817.

Distribución ibérica.- G: Ría de Foz, Lugo (Ariño, 1987; Junoy \& Viéitez, 1990a, 1990b, 1992; Junoy, 1996; Parapar et al., 1996). I: Estuario del Bidasoa (Marquiegui \& Aguirrezabalaga, 2009).

\section{Género Novafabricia Fitzhugh, 1990 \\ Novafabricia infratorquata (Fitzhugh, 1983)}

Bick (2005): 142-147, figs. 5, 6; Cepeda \& Lattig, 2016: 373376 , figs. 2,3 .

Distribución ibérica.-A: Gerona (Bick, 2005). B: Ibiza (Bick, 2005). Islas Columbretes, Mallorca y Menorca (Cepeda \& Lattig, 2016).

Género Parafabricia Giangrande, Gambi, Micheli \& Kroeker, 2014

Parafabricia mazzellae Giangrande, Gambi, Micheli \& Kroeker, 2014

Cepeda \& Lattig, 2016: 376-379, figs. 4, 5.

Distribución ibérica.- B: Islas Columbretes (Cepeda \& Lattig, 2016). 
Género Pseudofabricia Cantone, 1972

Pseudofabricia aberrans Cantone, 1972

Cepeda \& Lattig, 2016: 379-381, figs. 6, 7.

Distribución ibérica.- B: Mallorca y Menorca (Cepeda \& Lattig, 2016).

Género Pseudofabriciola Fitzhugh, 1990

Pseudofabriciola cf. capensis (Monro, 1937)

Gil (2011): 827 .

Fabricia cf. capensis (Monro, 1937).- San Martín \& Viéitez (1982): 19-21, fig. 2.

Distribución ibérica.- B: Mallorca (San Martín \& Viéitez, 1982).

Comentarios.- La única cita de la especie para el ámbito ibero-balear fue considerada dudosa por sus propios autores y está basada en un único ejemplar, por lo que Gil (2011) propone que podría pertenecer a algunas de las especies descritas en el mar Mediterráneo en fecha posterior (Fitzhugh et al., 1994).

Pseudofabriciola filamentosa (Day, 1963)

Fabricia filamentosa Day, 1963.- Méndez \& Cardell (1996): 140, tab. 1; Soler et al. (1997): 14, tab. 2.

Distribución ibérica.- A: Costa de Barcelona (Méndez \& Cardell, 1996). B: Formentera (Soler et al., 1997).

\section{Agradecimientos}

Los autores quieren expresar su gratitud con los doctores Patricia Lattig y Guillermo San Martín por su ayuda y consejo en la elaboración de esta investigación, así como por el préstamo de parte de la bibliografía consultada. Asimismo, los autores quieren agradecer los comentarios y sugerencias de dos evaluadores anónimos, por su contribución a la mejora de la calidad del texto. Este trabajo es parte de los resultados del proyecto "Fauna Ibérica. Polychaeta VI: Palpata-Canalipalpata I (CGL2014-53332-C5-3-P)", financiado por la Dirección General de Investigación del Ministerio de Educación y Ciencia.

\section{Referencias}

Abbiati, M., Bianchi, C. N., Castelli, A., Giangrande, A. \& Lardicci, C., 1991. Distribution of polychaetes on hard substrates of the midlittoral-infralittoral transition zone, western Mediterranean. Ophelia Supplement, 5: 421-432.

Acero, M. I. \& San Martín, G., 1986. Poliquetos epibiontes del primer horizonte de algas fotófilas en la provincia de Cádiz y Málaga. Estudio faunístico comparado. Boletín de la Real Sociedad Española de Historia Natural (Sección Biológica), 82(1-4): 5-24.

Aguirrezabalaga, F., 1984. Contribución al estudio de los Anélidos Poliquetos de la Costa de Guipúzcoa. Munibe, 36: 119-130.
Alcázar, J. \& San Martín, G., 2016. Serpúlidos (Annelida, Serpulidae) colectados en la campaña oceanográfica "Fauna II" y catálogo actualizado de las especies iberobaleares de la familia Serpulidae. Graellsia, 72(2): e053. http://dx.doi.org/10.3989/graellsia.2016.v72.120

Alós, C., 1983. Anélidos poliquetos del Cabo de Creus. II. Hojas de Posidonia oceanica. Publicaciones del Departamento de Zoología, Barcelona, 9: 23-30.

Alós, C., 1984. Anélidos poliquetos del Cabo de Creus. I. Rizomas de Posidonia oceanica. Cuadernos Marisqueros, Publicaciones Técnicas, 7: 89-101.

Alós, C., 1988. Anélidos Poliquetos del Cabo de Creus (Alt Emporda). Tesis Doctoral inédita. Universidad de Barcelona. $838 \mathrm{pp}$.

Alós, C., Campoy, A. \& Pereira, F., 1982. Contribución al estudio de los anélidos poliquetos endobiontes de esponjas. In: Ros, J. \& Niell, F. X. (eds.). Actas del II Simposio Ibérico de Estudios del Bentos Marino, San Sebastián: 139-157.

Altuna, A., Romero, A., Sanz, A., Torres Gómez de Cádiz, J. A. \& Ibáñez, M., 1983. Contribución al conocimiento de la fauna marina de la costa de Guipúzcoa I. Lurralde, 1983: 127-155.

Amoureux, L., 1972. Annélides Polychètes recueillies sur les pentes du talus continental, au large de la Galice (Espagne). Campagnes 1967 et 1968 de la "Thalassa". Cahiers de Biologie Marine, 13: 63-89.

Amoureux, L., 1973. Annélides Polychètes recueillies sur les pentes du talus continental au nord de la côte espagnole, Campagne 1970 de la "Thalassa". Cahiers de Biologie Marine, 14(4): 429-452.

Amoureux, L., 1974. Annélides Polychètes recueillies sur les pentes du talus continental au nord-ouest de l'Espagne et du Portugal (Campagne 1972 de la "Thalassa"). Cuadernos de Ciencias Biológicas, 3: 121-154.

Amoureux, L., 1987. Annélides Polychètes du talus continental européen. Données complémentaires concernant les campagnes 1970-1971-1972 de la "Thalassa". Données globales sur l'ensemble des six campagnes (1967 à 1973). Cahiers de Biologie Marine, 28: 569-577.

Amoureux, L. \& Calvário, J., 1981. Annélides Polychètes du Portugal. Données nouvelles. Arquivos do Museu Bocage, serie B, 1(12): 145-156.

Anadón, N., 1981. Contribución al conocimiento de la fauna bentónica de Vigo. III. Estudio de los arrecifes de Sabellaria alveolata (L.) (Polychaeta, Sedentaria). Investigación Pesquera, 45(1): 105-122.

Arias, A., Giangrande, A., Gambi, M. C. \& Anadón, N., 2013. Biology and new records of the invasive species Branchiomma bairdi (Annelida: Sabellidae) in the Mediterranean Sea. Mediterranean Marine Science, 14/1: 162-171. http://dx.doi.org/10.12681/mms.363

Ariño, A., 1987. Optimización de los estudios faunísticos: Ensayo sobre poliquetos sedentarios ibéricos. Tesis Doctoral inédita. Universidad de Navarra. 900 pp.

Ballesteros, E., 1998. Addicions a la fauna d'invertebrats bentònics marins de l'Arxipelag de Cabrera (Illes Balears, Mediterrània Occidental). Bolletí de la Societat d' Història Natural de les Balears, 41: 41-48. 
Baratech, L. \& San Martín, G., 1987. Contribución al conocimiento de los Anélidos Poliquetos (Annelida: Polychaeta) de las costas andaluzas. Boletín del Instituto Español de Oceanografía, 4(2): 37-48.

Bellan, G., 1959. Campagnes de la "Calypso": Mer d'Alboran. 2. Annélides Polychètes. Annales de l'Institut Océanographique, 37(1): 315-342.

Bellan, G., 1960. Annélides Polychètes. Résultats Scientifiques de la Campagne du N.R.P. "Faial" dans les eaux cotières du Portugal (1957), 2: 1-31.

Bick, A., 2005. Redescription of Fabriciola tonerella Banse, 1959, and a new record of Novafabricia infratorquata (Fitzhugh, 1983) from the Mediterranean Sea, with a key for the Fabriciinae (Annelida: Polychaeta) of the Mediterranean Sea and the north-east Atlantic. Zoologischer Anzeiger, 244: 137-152. http://dx.doi. org/10.1016/j.jcz.2005.07.002

Bustamante, M., 2013. Using rocky invertebrates to assess environmental impact. Tesis Doctoral inédita. Universidad del País Vasco. Leioa. 155 pp.

Bustamante, M., Tajadura-Martín, F. J. \& Saiz-Salinas, J. I., 2007. Intertidal macrofaunal communities in an intensely polluted estuary. Environmental Monitoring and Assessment, 134: 397-410. http://dx.doi. org/10.1007/s10661-007-9631-4

Cacabelos, E., 2005. Cartografía bionómica de las poblaciones macrobentónicas de sustratos blandos de la Ensenada de San Simón (Galicia). Tesis Doctoral inédita. Universidade de Vigo. Vigo. 614 pp.

Cacabelos, E., Gestoso, L. \& Troncoso, J. S., 2009. Inventario de la macrofauna bentónica de sustratos blandos de la Ensenada de San Simón (NO España). Boletín de la Real Sociedad Española de Historia Natural (Sección Biológica), 103(1-4): 103-119.

Camp, J., 1976. Comunidades bentónicas de sustrato duro del litoral NE español. IV. Poliquetos. Investigación Pesquera, 40(2): 533-550.

Campoy, A., 1982. Fauna de España. Fauna de Anélidos Poliquetos de la Península Ibérica. EUNSA (Ediciones de la Universidad de Navarra, S.A.), serie Biológica. Pamplona. 781 pp.

Campoy, A. \& Alquézar, E., 1982. Anélidos poliquetos de las formaciones de Dendropoma petraeum (Monterosato) de las costas del sureste de España. In: Ros, J. \& Niell, F. X. (eds.). Actas del II Simposio Ibérico de Estudios del Bentos Marino, San Sebastián: 121-137.

Campoy, A. \& Jordana, R., 1978. Contribución a la fauna de los Anélidos Poliquetos de las costas españolas. Nota sobre una relación de especies capturadas en Blanes (Gerona) y Águilas (Murcia). Boletín de la Real Sociedad Española de Historia Natural (Sección Biológica), 76(1): 49-56.

Capa, M., Pons, J. \& Hutchings, P., 2013. Cryptic diversity, intraspecific phenetic plasticity and recent geographical translocations of Branchiomma (Sabellidae, Annelida). Zoologica Scripta, 42, 637-655. http://dx.doi. org/10.1111/zsc. 12028

Capaccioni-Azzati, R., 1987. Anélidos Poliquetos de la ensenada de los Alfaques (delta del Ebro, Mediterráneo Occidental). Tesis Doctoral inédita. Universitat de València. $533 \mathrm{pp}$.
Capaccioni-Azzati, R., García-Carrascosa, M. \& Rodríguez-Babio, C., 1990. Caracterización ecológica de la fauna de Anélidos Poliquetos de la ensenada de los Alfaques (delta del Ebro, Mediterráneo Occidental). In: Gallego, L. (ed.). Bentos VI. Bilbilis. Palma de Mallorca: 89-100.

Capaccioni-Azzati, R., Torres-Gavilá, F. J. \& Tena, J., 1992. Dos nuevos géneros de anélidos poliquetos para la península Ibérica: Neopseudocapitella Rullier \& Amoureux, 1979 y Demonax Kinberg, 1867. Thalassas, 9: 115-121.

Cardell, M. J. \& Gili, J. M., 1988. Distribution of a population of annelid polychaetes in the "trottoir" of the midlittoral zone on the coast of North-East Spain, Western Mediterranean. Marine Biology, 99: 83-92. https:// doi.org/10.1007/BF00644980

Carvalho, R. N., 1929. Catálogo da colecção de invertebrados de Portugal existente no Museu Zoológico da Universidade de Coimbra. Vermes. I. Polychaeta. Memórias e Estudos do Museu Zoológico da Universidade de Coimbra, Série I, 37: 1-16.

Carvalho, S., Pereira, P., Pereira, F., Pablo, H., Vale, C., \& Gaspar, M. B., 2011. Factors structuring temporal and spatial dynamics of macrobenthic communities in a eutrophic coastal lagoon (Obidos Lagoon, Portugal). Marine Environment Research, 71: 97-110. http:// dx.doi.org/10.1016/j.marenvres.2010.11.005

Castro, M. L. F. \& Viegas, M. C. M., 1981. Povoamentos intertidais do Estuário do Tejo. Estudo preliminar da fácies de Mytilus galloprovincialis Lamarck, 1818. Boletim da Sociedade Portuguesa de Ciências Naturais, 20: 71-81.

Ceberio, A., Martínez, J. \& Aguirrezabalaga, F., 1998. Presencia de Desdemona ornata Banse, 1957 (Polychaeta, Sabellidae) en las costas de la Península Ibérica, Golfo de Vizcaya. Munibe, 50: 37-41.

Cepeda, D. \& Lattig, P., 2016. A new species of Polycirridae (Annelida: Terebellida) and three new reports for Cantabrian and Mediterranean Seas. Cahiers de Biologie Marine, 57: 371-381.

Cepeda, D. \& Lattig, P., 2017. New reports and description of a new species of Sabellidae (Annelida: Polychaeta) for the Iberian Peninsula and Balearic Archipelago. Marine Biology Research, 13(8): 832-853. http:// dx.doi.org/10.1080/17451000.2017.1303576

Cepeda, D. \& Rodríguez-Flores, P., 2017. First record of the invasive worm Branchiomma bairdi (Annelida: Sabellidae) in the Balearic Sea. Journal of the Marine Biological Association of the United Kingdom Research, https://doi.org/10.1017/S0025315417001618

Chainho, P., Fernandes, A., Amorin, A., Ávila, S. P., Canninng-Clode, J., Castro, J. J., Costa, A. C., Costa, J. L., Cruz, T., Gollasch, S., Grazziotin-Soares, C., Melo, R., Micael, J., Parente, M. I., Semedo, J., Silva, T., Sobral, D., Sousa, M., Torres, P., Veloso, V. \& Costa, M. J., 2015. Non-indigenous species in Portuguese coastal areas, coastal lagoons, estuaries and islands. Estuarine, Coastal and Shelf Science, 167: 199-211. http://dx.doi. org/10.1016/j.ecss.2015.06.019

Cochrane, S. J., 2000. Taxonomy and Systematics of selected marine soft-bottom fan worms (Annelida: Polychaeta: 
Sabellidae). Tesis Doctoral Inédita. University of St. Andrews. 296 pp.

Costello, M. J., Bouchet, P., Boxshall, G., Fauchald, K., Gordon, D., Hoeksema, B. W., Poore, G. C. B., van Soest, R. B. M., Stöhr, S., Walter, T. C., Vanhoome, B., Decock, W. \& Appeltans, W., 2013. Global coordination and standardisation in marine biodiversity through the World Register of Marine Species (WoRMS) and related databases. PLoS ONE, 8(1):e51629. http:// dx.doi.org/10.1371/journal.pone.0051629

Desbruyères, D., Guille, A. \& Ramos, J. M., 1972. Bionomie benthique du Plateau continental de côte catalane espagnole. Vie et Milieu (B), 23(2): 335-363.

Dexter, D. M., 1992. Soft-bottom invertebrates of the Portuguese benthos. Boletim do Instituto Nacional de Investigação das Pescas, 17: 61-88.

El Haddad, M., Assadi, C., Tasso, V., Villaroya, L., Gallardo, F. J., Capaccioni Azzati, R., García-Carrascosa, A. M., Sáez, J. \& Monforte, F., 2007. Catálogo preliminar de especies no indígenas de la biota marina del Puerto de Valencia (Mediterráneo Occidental) y su potencial invasivo. Actas II Congreso Nacional sobre Especies Exóticas Invasoras "EEI 2006", León 2006: 202-224.

El Haddad, M., Capaccioni Azzati, R. \& García-Carrascosa, A.M., 2008. Branchiomma luctuosum (Polychaeta: Sabellidae): a non-indigenous species at Valencia Port (western Mediterranean Sea, Spain). Marine Biodiversity Records, 1: e61. http://dx.doi.org/10.1017/ S1755267207006604.

El-Haddad, M., Capaccioni Azzati, R. \& García-Carrascosa, A. M., 2013. Annotated checklist Capitellidae (Annelida, Polychaeta) from the Iberian Peninsula, Chafarinas, Balearic and Canary Islands. Graellsia, 69(1): 97-116. http://dx.doi.org/10.3989/graellsia.2013.v69.082

El Haddad, M., Tasso Bermell, V., Gilabert Carmona, J. A. \& Assadi García, C., 2012. The use of georeferenced underwater TV devices for the study of the exotic invasive species Branchiomma luctuosum (Grube, 1869) (Polychaeta, Sabellida) in ports of the Eastern Iberian coast (Western Mediterranean Sea). BioInvasions Records, 1(4): 277-281. http://dx.doi.org/10.3391/ bir.2012.1.4.06

Fitzhugh, K., 1989. A systematic revision of the SabellidaeCaobangiidae-Sabellongidae complex (Annelida: Polychaeta). Bulletin of the American Museum of Natural History, 192: 1-104.

Fitzhugh, K., 1991. Further revision of the Sabellidae subfamilies and cladistic relationships among the Fabriciinae (Annelida: Polychaeta). Zoological Journal of the Linnean Society, 102 (4): 305-332.

Fitzhugh, K., Giangrande, A. \& Simboura, N., 1994. New species of Pseudofabriciola Fitzhugh, 1990 (Polychaeta: Sabellidae: Fabriciinae), from the Mediterranean Sea. Zoological Journal of the Linnean Society, 110: 219-241. http://dx.doi.org/10.1111/j.1096-3642.1991. tb00004.x

García-Arberas, L. \& Rallo, A., 1994. Fauna béntica de los fondos de la fosa del Capbreton (Golfo de Vizcaya. Atlántico oriental): Anélidos poliquetos. Cuadernos de Investigación Biológica (Bilbao), 18: 71-83.
Giangrande, A., 1994. The genus Demonax (Polychaeta, Sabellidae) in the Mediterranean Sea, with description of D. tommasi n. sp. Bolletino di Zoologia, 61: 229-233.

Giangrande, A. \& Licciano, M., 2008. Revision of the species of Megalomma (Polychaeta, Sabellidae) from the Mediterranean Sea, with the description of M. messapicum n. sp. Italian Journal of Zoology, 75(2): 207-217.

Giangrande, A., Licciano, M. \& Wasson, B., 2015. Guide to identification of Sabellidae and Fabriciidae (Polychaeta) in north east Atlantic and Mediterranean waters. NMBAQC 2014 Taxonomic Workshop. Dove Marine Laboratory Press. Letchworth. 91 pp.

Giangrande, A., Montanaro, P. \& Castelli, A., 1999. On some Amphicorina (Polychaeta, Sabellidae) species from the Mediterranean coast, with the description of A. grahamensis. Italian Journal of Zoology, 66(2): 195-203.

Gil, J., 2011. The European fauna of Annelida Polychaeta. Tesis Doctoral inédita. Universidade de Lisboa. 1554 pp.

Gómez, J. J. \& San Martín, G., 1985. Estudio faunísticoecológico de las taxocenosis de Poliquetos y Moluscos de la playa de la "Ribeira" (Asturias). 1. Resultados faunísticos. Boletín del Instituto Español de Oceanografía, 2(3): 111-118.

Gràcia, F., Clamor, B., Jaume, D., Fornós, J.J., Uriz, M.J., Martin, D., Gil, J., Gracia, P., Febrer, M. \& Pons, G., 2005. La Cova des Coll (Felanitx, Mallorca): espeleogènesi, geomorfologia, hidrologia, sedimentologia, fauna i conservacio. ENDINS, 27: 140-186.

Guyonnet, B. \& Borg, D., 2015. Premier signalement de l'espèce introduite Euchone limnicola Reish, 1959 (Polychaeta: Sabellidae) sur les côtes françaises de la Mer du Nord (Grand Port Maritime de Dunkerque). An Aod - Les Cahiers Naturalistes de l'Observatoire Marin, 4(2): 15-23.

Hartman, O., 1959. Catalogue of the Polychaetous Annelids of the World. Parts 1 and 2. Allan Hancock Foundation Occasional Paper, 23: 1-628.

Hartmann-Schöder, G., 1977. Polychetes aus dem Sublitoral und Bathyal vor der portugiesischen und marokkanischen Küste Auswertung der Fahrt 8 (1967) von F.S. "Meteor". "Meteor" Forschung-Ergebnisse, D, 26: 65-99.

Hartmann-Schröder, G., 1979. Die Polychaeten der “Atlantischen Kuppenfahrt" von F.S. "Meteor" (Fahrt 9 c, 1967). 1. Proben aus Schleppgeräten. "Meteor" Forschung-Ergebnisse, D, 31: 63-90.

Henke J., 1972. Erganzende Bemerkungen zur Makrofauna der spanischen Sudküste. Zoologischer Anzeiger, 189: 348-351.

International Commission of Zoological Nomenclature, 1999. International code of zoological nomenclature. Fourth edition. The International Trust for Zoological Nomenclature, Londres, $106 \mathrm{p}$.

Iroso, I., 1921. Revisione dei Serpulidi e Sabellidi del Golfo di Napoli. Pubblicazioni della Stazione Zoologica di Napoli, 3: 47-91.

Johansson, K. E., 1927. Beiträge zur Kenntnis der Polychaeten-Familien Hermellidae, Sabellidae und Serpulidae. Zoologiska bidrag från Uppsala, 11: 1-184. 
Junoy, J., 1996. La Ría de Foz, Comunidades Bentónicas. Servicio de Publicaciones de la Diputación Provincial de Lugo. Lugo. 210 pp.

Junoy, J. \& Viéitez, J. M., 1990a. Manayunkia aestuarina (Bourne, 1883) (Polychaeta, Sabellidae), en la Ria de Foz (Galicia, España). Boletín de la Real Sociedad Española de Historia Natural (Sección Biológica), 86(1-4): 86-94.

Junoy, J. \& Viéitez, J. M., 1990b. Macrozoobenthic community structure in the Ría de Foz, an intertidal estuary (Galicia, Northwest Spain). Marine Biology, 107: 329-339.

Junoy, J. \& Viéitez, J. M., 1992. Macrofaunal abundance analyses in the Ría de Foz (Lugo, Northwest Spain). Cahiers de Biologie Marine, 33: 331-345.

Knight-Jones, P., 1983. Contributions to the taxonomy of Sabellidae (Polychaeta). Zoological Journal of the Linnean Society, 79: 245-295. http://dx.doi. org/10.1111/j.1096-3642.1983.tb01167.x

Knight-Jones, P., Darbyshire, T., Petersen, M. E. \& TovarHernández, M. A., 2017. What is Pseudopotamilla reniformis (Sabellidae)? Comparisons of populations from Britain, Iceland and Canada with comments on Eudistylia and Schizobranchia. Zootaxa, 4254(2): 201-220. http://dx.doi.org/10.11646/zootaxa.4254.2.3

Knight-Jones, P., Knight-Jones, W. \& Ergen, Z., 1991. Sabelliform polychaetes, mostly from Turkey's Aegean coast. Journal of Natural History, 25: 837-858. http:// dx.doi.org/10.1080/00222939100770561

Knight-Jones, P. \& Perkins, T. H., 1998. A revision of Sabella, Bispira and Stylomma (Polychaeta: Sabellidae). Zoological Journal of the Linnean Society, 123: 385-467. http://dx.doi.org/10.1111/j.1096-3642.1998. tb01370.x

Kupriyanova, E. K. \& Rouse, G. W., 2008. Yet another example of paraphyly in Annelida: Molecular evidence that Sabellidae contains Serpulidae. Molecular Phylogenetics and Evolution, 46(3): 1174-1181. http://dx.doi. org/10.1016/j.ympev.2007.10.025

Laborda, A., Cimas, E. \& Marcilla, I., 1991. Influencia de la contaminación en la distribución de los anélidos poliquetos del sustrato rocoso intermareal de la región del cabo de Peñas (Asturias, España). Boletín del Instituto Español de Oceanografía, 7(1): 75-90.

Lo Bianco, S., 1893. Gli annelidi tubicoli nel Golfo di Napoli. Atti della Reale Accademie della Scienze fisiche e matematique, Serie 2, 5(44): 1-97.

López, E., 1995. Anélidos poliquetos de sustratos duros de las islas Chafarinas. Tesis Doctoral Inédita. Universidad Autónoma de Madrid. 672 pp.

López, E. \& Tena, J., 1999. A new species of Amphicorina (Polychaeta: Sabellidae: Sabellinae) from the Chafarinas Islands (Western Mediterranean). Cahiers de Biologie Marine, 40: 329-335.

López, E. \& Viéitez, J. M., 1999. Polychaete assemblages on non-encrusting algae from the Chafarinas Islands (W Mediterranean). Cahiers de Biologie Marine, 40: 375-384.

López-Jamar, E. \& González, G., 1987. Infaunal macrobenthos of the Galician Continental shelf off La Coruña
Bay, Northwest Spain. Biological Oceanography, 4(2): 165-192.

Lourido, A., 2009. Distribución espacial de la macrofauna bentónica de sustratos sedimentarios de la ría de Aldán (Galicia, NO de España). Tesis Doctoral inédita. Universidade de Vigo. Vigo. 483 pp.

Lourido, A., Cacabelos, E. \& Troncoso, J. S., 2008. Patterns of distribution of the polychaete fauna in subtidal soft sediments of the Ría de Aldán (north-west Spain). Journal of the Marine Biological Association of the United Kingdom, 88(2): 263-275. http://dx.doi.org/10.1017/ S0025315408000696

Malonda, I., 2008. Estudio de la taxocenosis de poliquetos como parte de la evaluación de la calidad ambiental en el puerto de Cartagena (Murcia, España). Tesis Doctoral inédita. Universidad Autónoma de Madrid. 289 pp.

Marquiegui, M. A. \& Aguirrezabalaga, F., 2009. Colonization process by microbenthic infauna after a managed coastal realignment in the Bidasoa estuary (Bay of Biscay, NE Atlantic). Estuarine, Coastal and Shelf Science, 84: 598-604.

Martin, D., 1987. La comunidad de Anélidos Poliquetos de las concreciones de algas calcáreas del litoral catalán. Caracterización de las especies. Publicaciones del Departamento de Zoología. Universidad de Barcelona, 13: 45-54.

Martin, D. \& Giangrande, A., 1991. Novafabricia bilobata sp. nov. (Polychaeta, Sabellidae, Fabriciinae) from the Mediterranean. Ophelia, 33: 113-120. http://dx.doi.org/ 10.1080/00785326.1991.10429734

Martin, D., Pinedo, S. \& Sardà, R., 2000. Distribution patterns and trophic structure of soft-bottom polychaete assemblages in a North-western Mediterranean shallow-water bay. Ophelia, 53: 1-17. http://dx.doi.org/10 .1080/00785326.2000.10409431

Martínez, J. \& Adarraga, I., 2001. Nuevas citas de anélidos poliquetos y de un anfípodo marino en la Península Ibérica. Boletín de la Real Sociedad Española de Historia Natural (Sección Biológica), 96(3-4): 137-150.

Martins, R., Sampaio, L., Rodrigues A. M. \& Quintino, V., 2013. Soft-bottom Portuguese continental shelf polychaetes: Diversity and distribution. Journal of Marine Systems, 123-124: 41-54. http://dx.doi.org/10.1016/j. jmarsys.2013.04.008

Méndez, N. \& Cardell, M. J., 1996. Littoral annelid polychaetes inhabiting soft bottoms of the Barcelonès (Catalonia, NE Spain). Miscel-lània Zoològica, 19.1: 119-147.

Monteiro-Marques, V., 1979. Contribution a l'étude du plateau continental des côtes portugaises (Algarve, Sud Portugal): étude des peuplements de substrats meubles. Téthys, 9(2): 191-195.

Monteiro-Marques, V., Sousa-Reis, C., Calvário, J., Marques, J. C., Melor, R., \& Santos, R., 1982. Contribução para o estudo dos povoamentos bentónicos (substrato rochoso) da costa ocidental portuguesa. Zona intertidal. Oecologia Aquatica, 6: 119-145.

Moreira, J., 2003. La fauna bentónica de la Ensenada de Baiona (Galicia, NO Península Ibérica): Diversidad, análisis de las comunidades, dinámica de poblaciones 
y distribución vertical. Tesis Doctoral inédita. Universidad de Vigo. 676 pp.

Moreira, J., Quintas, P. \& Troncoso, J. S., 2006. Spatial distribution of soft-bottom polychaete annelids in the Ensenada de Baiona (Ría de Vigo, Galicia, north-west Spain). Scientia Marina 70S3, 217-224.

Moreira, J. \& Troncoso, J. S., 2007. Inventario de la macrofauna bentónica de sedimentos submareales de la Ensenada de Baiona (Galicia, NO Península Ibérica). Nova Acta Cientifica Compostelana (Bioloxía), 16: 101-128.

Mucha, A. P. \& Costa, M. H., 1999. Macrozoobenthic community structure in two Portuguese estuaries: relationship with organic enrichment and nutrient gradients. Acta Oecologica, 20(4): 363-376. http://dx.doi. org/10.1016/S1146-609X(99)00130-7

Nobre, A. 1937. Fauna Marinha de Portugal. $1^{\circ}$ Aditamento. Memórias e Estudos do Museu Zoológico da Universidade de Coimbra, Série I, 93: 1-30.

Occhipinti-Ambrogi, A., Marchini, A., Cantone, G., Castelli, A., Chimenz, C., Cormaci, M., Froglia, C., Furnari, G., Gambi, M. C., Gianccone, G., Giangrande, A., Gravili, C., Mastrototaro, F., Mazziotti, C., Orsi-Relini, L. \& Piraino, S., 2011. Alien species along the Italian coasts: an overview. Biological Invasions, 13(1): 215237. http://dx.doi.org/10.1007/s10530-010-9803-y

Parapar, J., 1991. Anélidos Poliquetos bentónicos de la ría de Ferrol (Galicia). Tesis Doctoral inédita. Universidad de Santiago de Compostela. 1104 pp.

Parapar, J., Besteiro, C. \& Urgorri, V., 1992. Nuevas aportaciones al conocimiento de los anélidos poliquetos en el litoral gallego (N.O. Península Ibérica). Nova Acta Cientifica Compostelana (Bioloxía), 3: 109-123.

Parapar, J., Besteiro, C. \& Urgorri, V., 1996. Inventario dos poliquetos de Galicia (Annelida: Polychaeta). Cadernos da Área de Ciencias Biolóxicas, Inventarios, 16: 1-178.

Parapar, J., Martínez-Ansemil, E., Caramelo, C., Collado, R. \& Schmelz, R., 2009. Polychaetes and oligochaetes associated with intertidal rocky shores in a semi-enclosed industrial and urban embayment, with the description of two new species. Helgoland Marine Research, 63: 293-308. http://dx.doi.org/10.1007/s10152-009-0158-7

Pardal, M. A., Marques J.-C. \& Bellan, G., 1993. Spatial distribution and seasonal variation of subtidal polychaete populations in the Mondego estuary (western Portugal). Cahiers de Biologie Marine, 34: 497-512.

Perkins, T. H., 1984. Revision of Demonax Kinberg, Hypsicomus Grube, and Notaulax Tauber with a review of Megalomma Johansson from Florida (Polychaeta: Sabellidae). Proceedings of the Biological Society of Washington, 97: 285-368.

Pinedo, S., Sardà, R. \& Martin, D., 1999. Seasonal dynamics and structure of soft-bottom assemblages in Blanes Bay (northwest Mediterranean Sea). Publicaciones Especiales. Instituto Español de Oceanografía, 22: 61-70.

Quintas, P., 2005. Distribución espacial y temporal de los moluscos y anélidos poliquetos asociados a las praderas de Zostera marina L. y Zostera noltii Hornem. en la Ensenada de O Grove (Galicia, España). Tesis Doctoral inédita. Universidade de Vigo. Vigo. 488 pp.
Quintas, P., Cacabelos, E. \& Troncoso, J.S., 2012. Inventario de los moluscos y poliquetos asociados a las praderas de Zostera marina y Zostera noltei de la Ensenada de O Grove (Galicia, N-O España). Boletín de la Real Sociedad Española de Historia Natural (Sección Biológica), 106: 113-126.

Rallo, A., García-Arberas, L. \& Isasi, I., 1993. Fauna macrobentónica de los fondos del cañón de Capbretón: análisis faunístico de poliquetos, crustáceos y cnidarios y caracterización de puntos de muestreos según estos descriptores. Cahiers de Biologie Marine, 35: 69-90.

Ravara, A. \& Moreira, M. H., 2013. Polychaeta (Annelida) from the continental shelf off Aveiro (NW Portugal): Species composition and community structure. CheckList, 9(3): 533-539. http://dx.doi.org/10.15560/9.3.533

Redondo, M.S. \& San Martín, G., 1997. Anélidos poliquetos de la costa comprendida entre el cabo de San Antonio y el puerto de Valencia (Mediterráneo occidental). Publicaciones Especiales, Instituto Español de Oceanografía, 23: 225-233.

Reish, D. J., 1959. A new species of Sabellidae (Annelida, Polychaeta) from southern California. Annals and Magazine of Natural History, Series 13, 2: 717-719. http:// dx.doi.org/10.1080/00222935908655757

Rioja, E., 1916. Nota de algunos anélidos recogidos en las costas de Gijón y San Vicente de la Barquera. Boletín de la Real Sociedad Española de Historia Natural, 16: 462-466.

Rioja, E., 1917a. Datos para el conocimiento de a la fauna de Anélidos Poliquetos del Cantábrico. Trabajos del Museo Nacional de Ciencias Naturales de Madrid, serie Zoológica 29: 5-111.

Rioja, E., 1917b. Nota sobre algunos anélidos poliquetos recogidos en Málaga. Boletín de la Real Sociedad Española de Historia Natural, 17: 176-185.

Rioja, E., 1917c. Nota sobre una excursión por las costas de Gijón. Boletín de la Real Sociedad Española de Historia Natural, 17: 488-494.

Rioja, E., 1919. Adiciones a la fauna de Anélidos del Cantábrico. Revista de la Real Academia de Ciencias Exactas, Físicas y Naturales de Madrid, 17: 1-26.

Rioja, E., 1920. Una campaña biológica en el golfo de Valencia. Anales del Instituto General y Técnico de Valencia, 20: 1-36.

Rioja, E., 1923. Estudio sistemático de las especies ibéricas del suborden Sabelliformia. Trabajos del Museo Nacional de Ciencias Naturales de Madrid, serie Zoológica 48: 7-143.

Rioja, E., 1925. Anélidos Poliquetos de San Vicente de la Barquera (Cantábrico). Trabajos del Museo Nacional de Ciencias Naturales de Madrid, serie Zoológica 53: 5-62.

Rioja, E., 1931. Estudio de los Anélidos Poliquetos de la Península Ibérica. Memorias de la Academia de Ciencias Exactas, Físicas y Naturales de Madrid, serie Zoológica, 2: 1-471.

Rioja, E., 1935. Anélidos poliquetos procedentes de las campañas del Instituto Español de Oceanografía. Trabajos del Instituto Español de Oceanografía, 13: 1-41. 
Rodríguez, C. V. \& Viéitez, J. M., 1992. Macrofauna bentónica de los primeros metros del piso infralitoral de la costa de Punta Umbría (Huelva). Boletín del Instituto Español de Oceanografía, 82(2): 327-338.

Román, S., Pérez-Ruzafa, A. \& López, E., 2009. First record in the western Mediterranean Sea of Branchiomma boholense (Grube, 1878) (Polychaeta: Sabellidae), an alien species of Indo-Pacific origin. Cahiers de Biologie Marine, 50: 241-250.

Rouse, G. W. \& Pleijel, F., 2001. Polychaetes. Oxford University Press. Oxford. 354 pp.

Saldanha, L., 1974. Estudo do povoamento dos horizontes superiors da rocha litoral da costa da Arrabida (Portugal). Arquivos do Museu Bocage, $2^{a}$ série, 5(1): 1-382.

San Martín, G., Acero, M I., Contonente, M. \& Gómez, J. J., 1982. Una colección de anélidos poliquetos de las costas mediterráneas andaluzas. In: Ros, J. \& Niell, F. X. (eds.). Actas del II Simposio Ibérico de Estudios del Bentos Marino, San Sebastián: 171-182.

San Martín, G. \& Aguirre, O., 1991. Ciclo anual de los poliquetos asociados al alga Mesophyllum lichenoides (Ellis) en una playa mediterránea. Boletín del Instituto Español de Oceanografía, 7(1): 157-170.

San Martín, G. \& Alvarado, R. 1982. Nota sobre poliquetos de la isla de Cabrera (Baleares). Boletín de la Real Sociedad Española de Historia Natural (Sección Biológica), 79: 221-234.

San Martín, G., Estapé, S., García-Ocejo, A., Gómez, C. \& Jiménez, P., 1990. Estudio de la taxocenosis de anélidos poliquetos de los rizomas de Posidonia oceanica en las costas de Almería. Boletín del Instituto Español de Oceanografia, 6(1): 41-58.

San Martín, G. \& Viéitez, J. M., 1982. Contribución al estudio de la fauna de anélidos poliquetos de las costas españolas: Notas sobre dos poliquetos sedentarios (Ctenodrilus serratus y Fabricia cf. capensis) de la bahía de Palma de Mallorca. Boletín de la Real Sociedad Española de Historia Natural (Sección Biológica), 80: $17-21$.

San Martín, G. \& Viéitez, J. M., 1984. Anélidos Poliquetos de los rizomas de Posidonia oceanica en las costas de Cabo de Palos (Murcia, España). In: Boudouresque, C. F., Jeudy de Grissacm, A. \& Olivier, J. (eds.). Workshop Posidonia oceanica beds, 1: 149-157.

Sardà, R., 1982. Anélidos poliquetos de la bahía de Algeciras. Nota sobre una colección próxima a la desembocadura del río Guadarranque. In: Ros, J. \& Niell, F. X. (eds.). Actas del II Simposio Ibérico de Estudios del Bentos Marino, San Sebastián: 165-170.

Sardà, R., 1984. Estudio sobre los Poliquetos de las zonas mediolitoral e infralitoral en la región del Estrecho de Gibraltar. Tesis Doctoral inédita. Universidad de Barcelona. $901 \mathrm{pp}$.

Sardà, R., 1986a. Fauna de Anélidos Poliquetos de la región del Estrecho de Gibraltar, III-Eunicida, Orbiniida, Spionida, Magelonida, Chaetopterida, Ctenodrilida, Flabelligerida, Opheliida, Oweniida, Capitellida, Terebellida, Sabellida y Nerillida. Miscel·lània Zoològica, 10: 71-85.

Sardà, R., 1986b. Contribución al conocimiento de las poblaciones anelididanas infaunales de la costa catalana.
Publicaciones del Departamento de Zoología, Barcelona, 12: 27-36.

Sardà, R., 1991. Polychaete communities related to plant covering in the mediolittoral and infralitoral zones of the Balearic Islands (Western Mediterranean). P.S.Z.N. Marine Ecology, 12(4): 341-360.

Sardà, R., Pinedo, S. \& Martín, D., 2000. Seasonal dynamics of macroinfaunal key species inhabiting shallow softbottoms in the Bay of Blanes (NW Mediterranean). Acta Oecologica, 20: 315-326. http://dx.doi.org/10.1016/ S1146-609X(99)00135-6

Sardà, R., Serrano, L., Labrune, C., Gil, J., March, D., Amouroux, J. M., Taboada, S., Bonifacio, P. \& Grémare, A., 2014. Shallow-water polychaete assemblages in the northwestern Mediterranean Sea and its possible use in the evaluation of good environmental state. Memoirs of Museum Victoria, 71: 289-301. http://dx.doi. org/10.24199/j.mmv.2014.71.22

Serrano, A., 2002. Ecología de las poblaciones de poliquetos del entorno de la isla de Mouro (Santander, mar Cantábrico). Tesis Doctoral inédita. Universidad Autónoma de Madrid. 439 pp.

Serrano, A. \& Preciado, I., 2007. Environmental factors structuring polychaete communities in shallow rocky habitats: role of physical stress versus habitat complexity. Helgoland Marine Research, 61: 17-29. http:// dx.doi.org/10.1007/s10152-006-0050-7

Serrano, L. G., Cardell, M. J., Lozoya, J. P. \& Sardà, R., 2011. A polychaete-dominated community in the NW Mediterranean Sea, 20 years after cessation of sewage discharges. Italian Journal of Zoology, 78: 333-346. http://dx.doi.org/10.1080/11250003.2011.582298

Smith, R. S., 1991. Relationships within the order Sabellida (Polychaeta). Ophelia Supplement, 5: 249-260.

Sola Eslava, J. C., 1996. Listado de especies faunísticas y estudios de interés ecológico realizados en el estuario del Bidasoa (golfo de Vizcaya). Ozeanografika, 1: 27-41

Soler, A., Ballesteros, M. \& Turón, X., 1997. Poliquetos del Estany des Peix (Formentera, Baleares). Aproximación al estudio faunístico y ecológico. Historia Animalium, 3: 9-23.

Sousa-Reis, C., Monteiro-Marques, V., Calvário, J., Marques, J. C., Melo, R. \& Santos, R., 1982. Contribução para o estudo dos povoamentos bentónicos (substrato móvel) da costa ocidental portuguesa. Oecologia Aquatica, 6: 91-105.

Templado, J., Calvo, M., García Carrascosa, A. M., Boisset, F. \& Jiménez, J., 2002. Flora y fauna de la Reserva Marina de las islas Columbretes. Secretaría General de Pesca Marítima / Museo Nacional de Ciencias Naturales. Madrid. 263 pp.

Templado, J., Calvo, M., Moreno, D., Flores, A., Conde, F., Abad, R., Rubio, J., López-Fé, C. M. \& Ortiz, M., 2006. Flora y fauna de la Reserva Marina y Reserva de Pesca de la isla de Alborán. Secretaría General de Pesca Marítima / Museo Nacional de Ciencias Naturales. Madrid. 269 pp.

Templado, J., García-Carrascosa, M., Baratech, L., Capaccioni, R., Juan, A., López-Ibor, A., Silvestre, R. \& Massó, C., 1986. Estudio preliminar a la fauna asociada 
a los fondos coralíferos del Mar de Alborán (SE de España). Boletín del Instituto Español de Oceanografía, 3(4): 93-104.

Templado, J., Guerra, J., Bedoya, J., Moreno, D., Remón, J. M., Maldonado, M. \& Ramos, M. A. 1993. Fauna I. Fauna marina circalitoral del sur de la península Ibérica. Resultados de la campaña oceanográfica "Fauna I". Museo Nacional de Ciencias Naturales, CSIC. Madrid. 183 pp.

Tena, J., 1996. Faunística y ecología de los Anélidos Poliquetos de los fondos de sustrato duro del archipiélago de las Chafarinas (SW mar de Alborán). Tesis Doctoral inédita. Universitat de València. 549 pp.

Tena, J., Capaccioni-Azzati R., Torres Gavilá, F. J. \& García Carrascosa, A. M., 2000. Polychaetes associated with different facies of the photophylic algal community in the Chafarinas Archipelago (SW Mediterranean). Bulletin of Marine Science, 67(1): 55-72.

Tena, J., Capaccioni-Azzati R., Torres Gavilá, F. J. \& Porras, R., 1993. Anélidos poliquetos del antepuerto de Valencia: distribución y categorías tróficas. Publicaciones especiales. Instituto Español de Oceanografía, 11: 15-20.
Torres-Gavilá, F. J., 2008. Estudio faunístico, ecológico y ambiental de la fauna de Anélidos Poliquetos de sustratos sueltos de las islas Chafarinas (Mar de Alborán, S.W. Mediterráneo). Servei de Publicacions. Universitat de València. 695 pp.

Tovar-Hernández, M. A. \& Harris, L. H., 2010. Parasabella Bush, 1905, replacement name for the polychaete genus Demonax Kinberg, 1867 (Annelida, Polychaeta, Sabellidae). ZooKeys, 60: 13-19. http://dx.doi.org/10.3897/ zookeys.60.547

Tovar-Hernández, M. A., Licciano, M. \& Giangrande, A., 2007. Revision of Chone Krøyer, 1856 (Polychaeta: Sabellidae) from the Eastern central Atlantic and Mediterranean Sea with descriptions of two new species. Scientia Marina, 71(2): 315-338. http://dx.doi. org/10.3989/scimar.2007.71n2315

Zorita, I., Solaun, O., Galparsoro, I. \& Borja, A., 2009. Especies exóticas en el medio marino del País Vasco, en relación con el cambio global. Informe para la Dirección de Biodiversidad de la Viceconsejería de Medio Ambiente. Gobierno Vasco. Gobierno Vasco. Pasaia, $60 \mathrm{pp}$. 